\title{
La relocalisation des systèmes alimentaires dans les territoires : quel cadre d'analyse en ergonomie?
}

Une étude de cas sur le plateau de Saclay

Relocation of food systems within territories: which framework of analysis

in ergonomics? A case study in the Saclay plateau

Chloé Le Bail, Marianne Cerf et Gwenola Yannou-Le Bris

\section{(2) OpenEdition}

Journals

Édition électronique

URL : https://journals.openedition.org/activites/6980

DOI : $10.4000 /$ activites. 6980

ISSN : 1765-2723

Éditeur

ARPACT - Association Recherches et Pratiques sur les ACTivités

Référence électronique

Chloé Le Bail, Marianne Cerf et Gwenola Yannou-Le Bris, « La relocalisation des systèmes alimentaires dans les territoires : quel cadre d'analyse en ergonomie? », Activités [En ligne], 18-2 | 2021, mis en ligne le 15 octobre 2021, consulté le 07 avril 2022. URL : http://journals.openedition.org/activites/6980 DOI : https://doi.org/10.4000/activites.6980

Ce document a été généré automatiquement le 7 avril 2022.

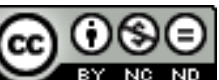

Activités est mis à disposition selon les termes de la licence Creative Commons Attribution - Pas d'Utilisation Commerciale - Pas de Modification 4.0 International. 


\section{La relocalisation des systèmes alimentaires dans les territoires : quel cadre d'analyse en ergonomie?}

Une étude de cas sur le plateau de Saclay

Relocation of food systems within territories: which framework of analysis

in ergonomics? A case study in the Saclay plateau

Chloé Le Bail, Marianne Cerf et Gwenola Yannou-Le Bris

\section{NOTE DE L'ÉDITEUR}

Article soumis le 26/02/2021, accepté le 09/07/2021

\section{Introduction}

Depuis quelques années maintenant, les ergonomes font part de leurs intérêts et de leurs préoccupations grandissantes concernant la prise en compte des enjeux du développement durable dans l'intervention. Une première perspective aborde ces enjeux à partir des activités de travail et des usages qui sont liés au développement durable. Par exemple, des problématiques apparaissent autour des thématiques du travail décent, de la mobilité verte, de l'habitat durable, ou encore de la pratique de l'agro-écologie (Guibourdenche, \& Cahour, 2019). Une deuxième perspective considère que la prise en compte des enjeux du développement durable implique un profond changement de la façon dont se réalisent des fonctions sociétales, par exemple l'alimentation. Cette dernière perspective invite à s'interroger sur la façon dont se sont organisées les chaînes de valeur pour remplir ces grandes fonctions, et sur la manière dont elles se reconfigurent et intègrent les enjeux du travail (Hubault, 2011 ; du Tertre, 2013). Dans les deux cas, un changement d'échelle d'analyse doit s'opérer : l'échelle de l'organisation n'est pas suffisante pour appréhender l'ampleur des projets de 
transformation des dimensions sociales, économiques et environnementales qui sont en œuvre (Boudra, Béguin, Delecroix, \& Pueyo, 2019 ; Thatcher, \& Yeow, 2016).

2 La crise sanitaire de la Covid-19 a mis en exergue la fragilité des chaînes de valeur mondialisées, notamment les vulnérabilités qu'induit la gestion en flux tendus de ces chaînes. Depuis, les politiques publiques affichent un regain d'intérêt pour la relocalisation de la production dans les territoires nationaux et notamment pour celle des filières alimentaires et agricoles. En septembre 2020, le plan France Relance a notamment prévu une enveloppe de trois millions d'euros réservée à l'émergence de nouveaux Projets Alimentaires Territoriaux (PAT) via l'appel à projets du Programme National pour l'Alimentation (PNA). Les principaux objectifs avancés sont de garantir l'autonomie et la sécurité alimentaire, de dynamiser l'économie locale, et de contribuer à réduire l'impact sur le climat des activités humaines. Cette réorganisation des chaînes de production et de distribution alimentaire ne peut s'entendre sans mutations du travail et de ce fait interpelle l'ergonomie.

3 Néanmoins, la relocalisation des systèmes alimentaires est bien antérieure à la crise sanitaire de la Covid-19. Depuis plusieurs années, on assiste à une prise de conscience à la fois politique et citoyenne de la déconnexion entre agriculture et alimentation. Cela se traduit par un regain d'intérêt des consommateurs pour les circuits alimentaires locaux et par l'essor de nouvelles formes de vente directe entre producteurs et consommateurs, telles que les $\mathrm{AMAP}^{1}$ ou les épiceries participatives (Lamine, \& Chiffoleau, 2016). Cette prise de conscience entraine des actions de la part d'acteurs divers, tels que les collectivités qui développent des PAT, ou encore les consommateurs qui demandent à manger plus durable et local, à leur domicile, mais aussi en restauration collective. Ces acteurs, par les projets qu'ils portent et parfois partagent, sont engagés dans des processus de reterritorialisation des systèmes alimentaires pour plus de durabilité.

4 Très peu d'études ont été conduites en ergonomie sur les activités susceptibles de contribuer à des systèmes alimentaires plus durables (Thatcher, Waterson, Todd, \& Moray, 2018). Comment l'ergonome peut-il envisager son intervention pour, d'une part analyser les enjeux du travail liés aux processus de reterritorialisation des systèmes alimentaires, et d'autre part contribuer à leur prise en compte dans la conception et le pilotage de ces processus? Ainsi, l'objectif de cet article est de proposer une approche ergonomique des processus de reterritorialisation des systèmes alimentaires pour fournir une alimentation locale et durable.

5 L'article repose sur une étude préliminaire que nous avons conduite sur le plateau de Saclay pour étudier l'approvisionnement de lieux de travail (cantine ou cafétéria d'entreprise) en produits locaux. L'étude de cas révèle une re-conception progressive et «dans l'usage » des situations de travail, des circuits alimentaires et des chaînes de valeur qui contribuent à l'approvisionnement des restaurants en produits locaux. Cette étude exploratoire nous invite à formuler une proposition conceptuelle pour appréhender ce que nous qualifions de travail de territorialisation. Ce travail sur lequel nous nous focalisons est celui qui permet à un système organisé pour assurer un approvisionnement en produits locaux, de fonctionner et de se développer. Dans la section 2, nous introduisons plusieurs acceptions du processus de reterritorialisation des systèmes alimentaires afin de dégager des éléments de compréhension pour envisager une approche ergonomique de ce processus. Nous présentons dans la section 3 notre étude de cas qui pointe les questions théoriques et méthodologiques 
pour aborder, d'un point de vue ergonomique, la reterritorialisation des systèmes alimentaires et ses impacts sur les activités des acteurs divers qui y prennent part. Dans la section 4, nous proposons alors un cadre d'analyse $\mathrm{du}$ processus de reterritorialisation des systèmes alimentaires et du travail de territorialisation.

\section{Comment aborder la reterritorialisation des systèmes alimentaires en ergonomie?}

6 Un système alimentaire se définit comme «un réseau interdépendant d'acteurs (entreprises, institutions financières, organismes publics et privés), localisé dans un espace géographique donné (région, état, espace multinational) et participant directement ou indirectement à la création de flux de biens et services orientés vers la satisfaction des besoins alimentaires d'un ou plusieurs groupes de consommateurs localement ou à l'extérieur de la zone considérée » (Rastoin, \& Ghersi, 2010, p. 556). On comprend dès lors que l'appellation « système alimentaire » recouvre des organisations qui s'étendent à des échelles variables (mondiale, nationale, locale), faisant intervenir des catégories d'acteurs et délivrant des offres de produits et de services propres à chacune de ces échelles. Qu'implique alors une approche des processus de territorialisation des systèmes alimentaires pour plus de durabilité?

\subsection{Systèmes alimentaires locaux et systèmes alimentaires territorialisés}

7 Des travaux en sociologie ou en économie se sont particulièrement intéressés à ces processus, en mobilisant les notions de systèmes alimentaires locaux (SAL ; Muchnick, Requier-Desjardins, Sautier, \& Touzard, 2007) ou systèmes alimentaires territorialisés (SAT ; Lamine, 2012). L'une et l'autre s'inscrivent dans des travaux qui s'intéressent aux dynamiques d'innovation. La première notion (SAL) trouve son origine dans les travaux sur les systèmes productifs locaux (Porter, 1998) et a émergé à partir de travaux menés en Amérique Latine ou en France sur les produits sous signe de qualité. Les auteurs qui s'y réfèrent ne partagent pas tous la même façon d'aborder les activités agroalimentaires et leurs liens au territoire. Ainsi, pour certains, ces activités sont analysées comme une composante d'un ensemble socioéconomique dont l'espace concret est postulé ex ante (par exemple, une région administrative). Pour d'autres, le périmètre du territoire (ou certaines de ses composantes) découle des actions et formes prises par l'activité agroalimentaire, considérée alors comme " localisée » et non locale. Dans ce cas, l'ancrage territorial des activités agroalimentaires est lié à aux actions locales de coordination entre des activités, et l'accent est mis sur la dimension collective, locale et singulière des liens physiques, cognitifs, historiques, interpersonnels et institutionnels entre activités qui se concrétisent dans des biens publics locaux, des institutions, des réseaux, et des connaissances partagées et distribuées.

8 La seconde notion (SAT) voit le jour plus récemment en lien avec les cadres développés sur les transitions vers la durabilité (Geels, \& Schot, 2007) dans une perspective sociohistorique, et avec les approches des transitions au niveau très fin des trajectoires professionnelles des agriculteurs (Cardona, Lamine, \& Hochereau, 2012 ; Chantre, \& Cardona, 2014). Ainsi, Lamine (2012, p. 139) définit les systèmes alimentaires 
territorialisés comme des systèmes «englobant non seulement les acteurs locaux des filières de production, de transformation, de distribution, mais aussi le conseil technique, les politiques publiques territoriales ou territorialisées, les consommateurs et la société civile. On peut dire que ce système englobe également, de fait, les différents dispositifs et réseaux mettant en relation production, commercialisation et consommation (qu'il s'agisse de circuits courts ou longs)». Alors que la notion de SAL a surtout permis d'éclairer la construction dans le temps de l'ancrage territorial, la notion de SAT s'intéresse à la façon dont s'agrègent différents systèmes agroalimentaires alternatifs et profitent de fenêtres d'opportunité pour bousculer le système alimentaire dominant dans un espace géographique donné qui fait territoire pour les acteurs de ces systèmes alternatifs.

9 Ces travaux montrent que la reterritorialisation repose sur la structuration de réseaux d'acteurs impliqués dans les systèmes alimentaires (Lamine, \& Chiffoleau, 2016) qui s'auto-organisent au sein d'un nouveau territoire qu'ils dessinent pour répondre à leurs attentes qui ne sont pas satisfaites par le fonctionnement dominant. Développées par des disciplines comme la sociologie, l'économie et la géographie, et parfois dans le cadre de travaux interdisciplinaires associant des agronomes ou des technologues des aliments, certaines recherches abordent les pratiques agricoles ou artisanales tout en étant attentives aux pratiques de coordination qui permettent la construction, le maintien ou le déploiement de ces systèmes alimentaires locaux ou territorialisés. Néanmoins, le regard porté aborde peu les mutations que ces pratiques impliquent dans la réalisation du travail quotidien pour les travailleurs. L'approche ergonomique pourrait ainsi compléter ces travaux.

\subsection{Une proposition d'approche ergonomique qui mobilise les sciences de gestion}

10 Appréhender la reterritorialisation des systèmes alimentaires d'un point de vue ergonomique suppose néanmoins de délimiter ce qui "fait territoire» pour l'ergonomie. Tout comme les disciplines qui lui sont proches - la sociologie, l'anthropologie, et l'ethnologie - l'ergonomie se heurte à la difficulté de définir la notion de territoire, tant cet objet est complexe et polysémique (Boudra et al., 2019; Marié, 2004).

11 Un premier point de vue consiste à articuler d'une part le cadre pour l'action publique, la représentation politique et l'administration locale, et d'autre part «les diverses formes de rapport à l'espace que les individus et les groupes sociaux ne cessent de produire et de transformer dans le cadre de leurs relations sociales " (Alphandéry, \& Bergues, 2004, p. 5). Cette articulation rappelle notamment celle qui existe à l'échelle des entreprises et des organisations, entre d'une part le travail qui est prescrit par les acteurs décisionnaires, et d'autre part les formes d'interaction et de coopération qui s'établissent dans le travail réel. Un autre point de vue consiste à appréhender le territoire comme un processus, dont le façonnement résulte des activités de vie et de travail visant à définir un bien commun; de ce fait le territoire est aussi le révélateur de ces activités (Cunha, à paraître). Le territoire implique aussi des dimensions symboliques et culturelles qui rendent compte de l'attachement des acteurs à un espace physique donné, au sein duquel ils identifient des valeurs plus ou moins partagées et défendent des ressources (matérielles: terres, fleuves, abris, etc.; mais aussi 
immatérielles: savoir-faire, langage vernaculaire, etc.) plus ou moins communes (Pesqueux, 2014). Enfin, le territoire inclut une dimension géographique qui souligne l'existence d'un espace de référence situé à l'intérieur de frontières naturelles ou définies par les acteurs qui y vivent (Ibid.).

Nous proposons d'appréhender la reterritorialisation des systèmes alimentaires en considérant le territoire comme un système ouvert, dynamique et situé, qui se structure dans le temps et l'espace au gré des activités humaines, des interactions sociales entre les individus et les organisations, et des interactions entre l'Homme et son milieu naturel (Flores, \& Medeiros, 2018). Comme tout système complexe, le territoire est mouvant et évolutif (Moine, 2006), et constitué d'éléments organisés par le cadre législatif et politique s'instanciant au sein d'un espace géographique, mais aussi d'interactions fondées sur les multiples choix ou volontés des acteurs présents sur un espace, qui réorganisent la gestion et l'aménagement de cet espace.

Dans la suite de l'article, nous présentons une étude de cas exploratoire qui s'est construite à l'interface entre sciences de gestion et ergonomie. Nous avons mobilisé les sciences de gestion pour appréhender les transformations organisationnelles qui se jouent dans la reterritorialisation des systèmes alimentaires. En effet, la construction de l'organisation constitue l'objet d'étude des sciences de gestion qui proposent des modèles structurants des organisations (par exemple, Livian, 1998), dont l'instanciation dépend notamment de l'analyse des flux (de matière, d'information, et financiers) qui parcourent ces organisations, mais aussi des outils de gestion qui sont mobilisés pour les piloter (Hatchuel, \& Weil, 1992). Ces analyses visent à émettre des préconisations afin d'accroitre la performance de l'organisation, c'est-à-dire de rapprocher ses résultats de ses objectifs, quelle que soit la nature de ces objectifs ${ }^{2}$. Nous combinons ce regard avec l'approche ergonomique développée pour étudier la coordination intermétiers dans la relation de service (Arnoud, 2013 ; Caroly, \& Weill-Fassina, 2007 ; Motté, \& Haradji, 2010). Ainsi, il s'agit de saisir ces coordinations comme une activité collective distribuée dans le temps et l'espace, et qui traverse les entités organisationnelles pour produire des services de restauration ou de distribution alimentaire. Comme toute activité collective sous-entend la répartition de tâches, il s'agit donc de porter l'attention sur la coordination des activités individuelles et la réélaboration de règles pour gérer les conflits de logiques et garantir un travail «bien fait» (Caroly, 2010; Leplat, 1993).

\section{3. Étude de cas : l'approvisionnement de lieux de travail en produits locaux sur le plateau de Saclay}

14 Nous avons ainsi réalisé une première étude de cas exploratoire (Yin, 2003) pour établir un cadre d'analyse à développer ultérieurement. Le point de départ de notre étude est l'approvisionnement de lieux de travail (cantine ou cafétéria des entreprises privées et des organismes publics) en produits locaux sur le plateau de Saclay, un territoire agriurbain de la région île-de-France.

\subsection{Contexte de l'étude}

15 Le plateau de Saclay est un territoire en pleine mutation où s'entremêlent des enjeux liés à l'urbanisation et au développement économique (installation d'entreprises 
privées et d'infrastructures publiques, notamment une université et des grandes écoles), des enjeux de maintien d'une activité agricole (diminution des terres agricoles avec l'urbanisation), des enjeux de gestion des ressources naturelles, et des enjeux de gouvernance territoriale puisque qu'il implique la fusion de plusieurs communautés d'agglomération (Petit, Levavasseur, \& Verger, 2018). Le territoire administratif est la communauté d'agglomération Paris-Saclay qui regroupe 27 communes de l'Essonne. Le territoire géographique est à cheval sur les départements des Yvelines et de l'Essonne. Une Zone de Protection Naturelle Agricole et Forestière (ZPNAF) a été créée et inscrite dans la loi du Grand Paris du 3 juin 2010, aboutissant à ce que 2469 hectares de terres soient sanctuarisés pour l'activité agricole.

Le plateau de Saclay est au cœur d'un Projet Alimentaire Territorial ${ }^{3}$ (PAT), le premier à avoir vu le jour en Île-de-France. Ce projet concerne 58 communes, trois communautés d'agglomération, et est animé en partie par une association locale qui œuvre pour le maintien d'un équilibre solide entre monde agricole et monde urbain. Ce PAT déborde les frontières du territoire administratif de la communauté de Saclay pour inclure une partie du territoire de la communauté de Saint-Quentin en Yvelines et de Versailles Grand Parc.

Bien que l'association qui porte le PAT n'ait pas exprimé une demande particulière concernant l'approvisionnement en produits locaux des entreprises privées et publiques installées sur le plateau de Saclay, nous avons choisi de focaliser l'étude sur ce volet, peu pris en charge aujourd'hui par les acteurs des collectivités. En effet, si l'alimentation locale au travail s'inscrit dans le contexte plus général de l'alimentation durable en restauration collective, les entreprises privées ne sont pas encore concernées par la loi EGalim qui impose dès le $1^{\mathrm{er}}$ janvier $2022,50 \%$ de produits durables, dont $20 \%$ issus de l'agriculture biologique, en restauration collective dans tous les établissements de service public ${ }^{4}$. De plus, ces restaurants sont encouragés à s'approvisionner en produits obtenus dans le cadre de PAT bien que ces produits ne soient pas comptabilisés dans les $50 \%$ de produits durables.

\subsection{Recueil et analyse des données}

Le recueil des données a été mené entre octobre 2019 et mai 2020 par des chercheuses en ergonomie et en sciences de gestion. Nous avons utilisé la méthode des entretiens exploratoires pour comprendre ce qui est mis en place par les acteurs ${ }^{5} \mathrm{du}$ système alimentaire pour offrir des produits locaux et durables en restauration d'entreprise. Au total, 12 entretiens ont été menés (11 en face à face et un à distance, d'une durée moyenne d'une heure). Notre objectif était d'interroger une multiplicité d'acteurs avec des rôles et des points de vue divers, mais qui entendent tous participer au développement d'une alimentation durable et locale sur le plateau de Saclay. Plus précisément, nous avons interrogé les types d'acteurs suivants :

- Des experts nationaux et territoriaux de la fonction sociétale "alimentation » et de la restauration collective. L'objectif était d'identifier le fonctionnement générique de cette fonction et les leviers de changement à l'œuvre, les opérateurs traditionnels et les opérateurs émergents, le rôle des politiques publiques, et les changements globaux induits par la relocalisation des activités.

- Des acteurs locaux qui participent à la définition administrative, géographique ou culturelle du territoire. L'objectif était de saisir les spécificités du territoire et sa « structure » plus ou 
moins formelle qui guide les activités humaines. Nous avons interrogé l'association locale qui participe au projet de construction du cluster Paris-Saclay et qui défend la préservation et la valorisation des terres agricoles sur le territoire.

- Des acteurs locaux dont le travail quotidien contribue au développement d'une offre en alimentation locale dans le territoire administratif ou aux frontières de ce dernier. Nous avons notamment interrogé :

1. Des acteurs qui interviennent dans le développement d'une alimentation durable et locale dans des établissements privés et publics du plateau de Saclay. Lorsque l'établissement possédait une cantine ou une cafétéria, nous avons rencontré le responsable du restaurant ou bien la personne en charge du contrat avec la société prestataire du service de restauration ${ }^{6}$. Lorsque l'établissement ne possédait pas de cantine, nous avons rencontré la personne animatrice des problématiques du développement durable dans l'établissement. Les entretiens menés traitaient de : Comment et sous l'impulsion de quels facteurs se fait l'introduction d'une alimentation que ces acteurs qualifient de durable et/ou locale? Qu'estce que cela change dans leur travail de manager? Qu'est-ce que cela change dans le travail des employés de la restauration collective?

2. Des producteurs et/ou transformateurs de l'offre locale insérée dans les menus des restaurants enquêtés. Cette étape nous a permis d'explorer des chaînes d'approvisionnement locales, "de la fourchette (restaurants enquêtés) à la fourche (exploitations agricoles enquêtées) ${ }^{7} »$. Auprès des exploitants agricoles, nous avons notamment questionné : comment et sous l'impulsion de quels facteurs ont-ils orienté une partie de leur offre vers la restauration collective? Qu'est-ce que cela change dans leur travail de producteur et/ou de transformateur?

19 Le tableau 1 présente la typologie des acteurs interrogés et les organisations auxquelles ils appartiennent. Nous entendons ici par organisation un système composé de deux entités, d'une part une structure formelle qui guide les activités humaines ; d'autre part un groupe d'acteurs qui modifient cette structure à travers leurs interactions sociales et leurs activités (Reynaud, 1997). Ainsi, nous restituerons nos résultats en nous plaçant au niveau de l'organisation tout en évoquant certaines évolutions qui se font jour dans les situations de travail quotidiennes.

Pour des raisons d'anonymisation des données, nous ne présentons pas précisément les profils des personnes interrogées. Par ailleurs, il convient de préciser que nous n'avons pas accordé le même statut à tous les entretiens. Les entretiens 1 à 4 nous ont permis de saisir le cadre de l'action publique en matière d'alimentation, ainsi que le fonctionnement de la restauration et du plateau de Saclay. Les recoupements de leurs propos nous ont permis d'estimer que ces quatre entretiens étaient suffisants pour appréhender cet écosystème. Les entretiens 5 à 12 ont éclairé le travail réalisé au sein d'organisations qui participent à l'approvisionnement de certains restaurants collectifs en produits locaux. Ce sont ces entretiens qui ont été analysés finement. L'ensemble des entretiens nous a permis d'identifier un réseau d'organisations qui œuvrent pour le développement de filières alimentaires locales sur le plateau de Saclay. Comme le précise le tableau 1, chaque organisation étudiée peut être reliée à au moins une autre organisation étudiée, à l'exception de l'école d'ingénieur A. 
Tableau 1 : Typologie des acteurs interrogés et organisation d'appartenance. Table 1: typology of actors interviewed and their organization

\begin{tabular}{|c|c|c|}
\hline Acteur & Typologie & Organisation \\
\hline 1 & $\begin{array}{l}\text { Expert de l'agro- } \\
\text { alimentaire en Île- } \\
\text { de-France }\end{array}$ & $\begin{array}{l}\text { Organisme qui promeut des produits et savoir-faire agricoles, et qui soutient } \\
\text { des entreprises alimentaires franciliennes dans leurs projets de création ou } \\
\text { de développement. La ferme B est soutenue par cet organisme. }\end{array}$ \\
\hline 2 & $\begin{array}{l}\text { Expert de la } \\
\text { restauration } \\
\text { collective } 1\end{array}$ & $\begin{array}{l}\text { Organisme qui propose une démarche d'accompagnement au } \\
\text { développement d'une cuisine saine et respectueuse de l'environnement, en } \\
\text { restauration collective. L'Association C est insérée dans la démarche. }\end{array}$ \\
\hline 3 & $\begin{array}{l}\text { Expert de la } \\
\text { restauration } \\
\text { collective } 2\end{array}$ & $\begin{array}{l}\text { Réseau interprofessionnel de la restauration collective en gestion directe } \\
\text { pour representer la profession et accompagner les politiques alimentaires. } \\
\text { L'Association C fait partie de ce réseau. }\end{array}$ \\
\hline 4 & $\begin{array}{l}\text { Chargé de mission } \\
\text { chez Terre \& Cité } \\
\text { (association locale) }\end{array}$ & $\begin{array}{l}\text { Association qui oeuvre pour la protection de l'équilibre entre les espaces } \\
\text { ruraux et urbains sur le plateau de Saclay. Elle anime un espace d'échange } \\
\text { entre les agriculteurs et les autres acteurs du territoire. Les fermes A et B ont } \\
\text { crée l'association. }\end{array}$ \\
\hline 5 & $\begin{array}{l}\text { Responsable du } \\
\text { contrat avec la } \\
\text { société de services } \\
\text { de restauration }\end{array}$ & $\begin{array}{l}\text { Ecole d'ingénieur A : les salariés ont accès à une petite cafétéria avec une } \\
\text { offre durable très limitée, et dont la gestion est concédée à une sociêté de } \\
\text { services de restauration. }\end{array}$ \\
\hline 6 & $\begin{array}{l}\text { Responsable } \\
\text { développement } \\
\text { durable }\end{array}$ & $\begin{array}{l}\text { Ecole d'ingénieur B : les salariés ont accès à plusieurs restaurants } \\
\text { universitaires et cafétérias au sein de l'école, dont la gestion est confiée au } \\
\text { CROUS de Versailles. }\end{array}$ \\
\hline 7 & $\begin{array}{l}\text { Responsable de la } \\
\text { restauration }\end{array}$ & $\begin{array}{l}\text { Association C : elle gère trois restaurants et une sandwicherie (gestion } \\
\text { directe) pour le personnel universitaire. Les restaurants se fournissent chez } \\
\text { les fermes A et B. }\end{array}$ \\
\hline 8 & $\begin{array}{l}\text { Responsable des } \\
\text { contrats avec les } \\
\text { sociétés de services } \\
\text { de restauration }\end{array}$ & $\begin{array}{l}\text { Organisme D : les salariés ont accès à six restaurants, quatre sont internes à } \\
\text { l'organisme et sont gérés par une unique société de restauration; les deux } \\
\text { autres sont des Restaurants Inter-Entreprises gérés par une autre société de } \\
\text { restauration. Les restaurants proposent des produits de la ferme B. }\end{array}$ \\
\hline 9 & $\begin{array}{l}\text { Responsable d'un } \\
\text { restaurant }\end{array}$ & $\begin{array}{l}\text { Le CROUS de Versailles : compte plus de } 60 \text { structures de restauration. Le } \\
\text { restaurant enquêté sert entre } 200 \text { et } 800 \text { couverts par jour. Ce restaurant } \\
\text { propose des produits de la ferme B. }\end{array}$ \\
\hline 10 & $\begin{array}{l}\text { Exploitants } \\
\text { agricoles }\end{array}$ & $\begin{array}{l}\text { Ferme A : l'exploitation produit des céééales (maïs, blé, épeautre) et autres } \\
\text { cultures (colza, lentilles, pommes de terre...). Elle a converti une partie de } \\
\text { sa production à l'agriculture biologique. La ferme fabrique du pain Bio au } \\
\text { levain directement à partir du ble de la ferme. }\end{array}$ \\
\hline 11 & $\begin{array}{l}\text { Responsable de la } \\
\text { stratégie } \\
\text { commerciale }\end{array}$ & $\begin{array}{l}\text { Ferme B : I'exploitation est une ferme laitière de } 350 \text { vaches qui fabrique } \\
\text { des produits laitiers labellisés BLEU-BLANC-CEER. La laiterie fournit } \\
\text { des yaourts à plusieurs cantines du plateau de Saclay. }\end{array}$ \\
\hline 12 & Exploitant agricole & $\begin{array}{l}\text { Légumerie centrale qui n'est pas implantée sur le plateau de Saclay mais qui } \\
\text { fournit plusieurs acteurs de la restauration collective en région fle-de- } \\
\text { France, notamment les sociétés de services de restauration que l'on peut } \\
\text { retrouver sur Saclay. Les légumes des producteurs de la région sont } \\
\text { regroupés, transformés, conditionnés, puis redistribués dans les restaurants. }\end{array}$ \\
\hline
\end{tabular}

21 L'analyse des entretiens visait deux objectifs. D'abord, il s'agissait d'appréhender la manière dont les personnes interrogées articulent leurs activités avec celles d'autres acteurs pour fournir une alimentation qu'ils estiment durable et locale. Ensuite, il s'agissait d'identifier, à travers leurs discours, les effets que cet approvisionnement en produits locaux implique sur les activités de travail au sein des différentes entités organisationnelles (entreprises, fermes, restaurants).

Plus précisément, nous avons identifié la manière dont chaque acteur individuel engagé dans l'activité collective gère deux natures de coordination. La première est une coordination externe ou inter-organisationnelle (Fabbe-Costes, \& Lancini, 2009) qui relie l'entité organisationnelle à ses clients, ses fournisseurs et ses partenaires. À cet égard, l'analyse de la manière dont se structurent et évoluent les flux (délais, quantité, qualité, etc.) à la maille « organisation » s'est appuyée sur des approches des sciences de gestion (Hatchuel, \& Weil, 1992 ; Livian, 1998). La deuxième est une coordination interne ou intra-organisationnelle (Poret, Folcher, Motté, \& Haradji, 2016) qui permet à l'entité organisationnelle de piloter ses activités de travail. À ce titre, l'analyse de la manière dont les règles du travail sont prescrites et évoluent à la maille « situation de travail » repose sur les concepts de l'ergonomie.

De manière inductive et à l'aide de la littérature dans les deux champs disciplinaires, nous avons identifié des éléments qui déterminent les deux natures de coordination :

- Les règles de fonctionnement relatives à la coordination entre les travailleurs et/ou entre les différentes parties prenantes;

- Le pilotage de ces règles de coordination, c'est-à-dire les acteurs qui prennent part à la définition des règles et les modalités de prise de décision pour acter les évolutions des règles (démarche descendante versus participative, etc.) ; 
- Les flux d'information, qui renvoient à la manière dont circule l'information nécessaire à la coordination (modalités de recueil de l'information, de traitement, etc.) ;

- Les substrats formels de gestion, entendus comme les supports concrets qui soutiennent la coordination (contrats de collaboration, plannings, tableaux de référencement, etc.) ;

- Les ressources humaines, c'est-à-dire les moyens humains, les connaissances et les compétences nécessaires à la coordination ;

- Les ressources matérielles, c'est-à-dire le capital physique (techniques de production, équipements, accès aux matières premières) dont dispose l'organisation et qui rentre en jeu dans la manière dont se met en œuvre la coordination ;

- Le respect des délais, des quantités, de la qualité et du coût de revient ;

- Les normes de la restauration collective, qui comme pour toute filière, impose une certaine forme de culture entre les clients et les fournisseurs (attendus implicites, pratiques dominantes, démarches qualités précises, etc.), et est également contrainte par un cadre législatif (par exemple, normes sanitaires et d'hygiène);

- Les motivations et les aléas, entendus comme la part d'incertitude dans la coordination qui repose sur la subjectivité (valeurs et objectifs personnels, perception d'autrui).

Le tableau 2 présente la manière dont les entretiens ont été analysés. Ils ont d'abord été découpés en plusieurs séquences évoquant des motifs ou des étapes de coordination (par exemple, référencement du fournisseur, prise de commande, etc.). Puis une analyse sémantique a permis d'identifier les éléments en jeu dans ces étapes de coordination. L'idée principale était de rendre compte, d'une part de la dynamique entre les deux natures de coordination (interne et externe), et d'autre part des relations entre les différents éléments de coordination en jeu.

Tableau 2 : Analyse sémantique des entretiens, exemples. Table 2: semantic analysis of interviews, examples

\begin{tabular}{|c|c|c|}
\hline Exemple de séquence (titre puis verbatim) & $\begin{array}{l}\text { Coordination } \\
\text { interne }\end{array}$ & $\begin{array}{l}\text { Coordination } \\
\text { externe }\end{array}$ \\
\hline $\begin{array}{l}\text { Référencement / contractualisation : «Le responsable de la } \\
\text { restauration voulait du pain bio, } 100 \text { kilos par jour. Je lui ai dit, il n'y a } \\
\text { pas de problèmes Monsieur, je vais vous expliquer ce qu'il va se passer, } \\
\text { donc on embauche un boulanger puisque c'est une cuisson en soi ; je } \\
\text { lui dis vous avez combien de sites à livrer? Il me dit } 18 \text {; donc } \\
\text { j'embauche aussi un livreur et j'achète aussi une voiture. [...] Ce sont } \\
\text { des gens qui t'appellent et te disent je voudrais commander du pain, donc } \\
\text { nous dans notre organigramme on organise quelle journée on dédit } \\
\text { la quantité, puis après il te fait finalement une commande par mois » }\end{array}$ & $\begin{array}{l}\text { Ressources } \\
\text { humaines } \\
\text { Ressources } \\
\text { matérielles } \\
\text { Règles de } \\
\text { fonctionnement }\end{array}$ & $\begin{array}{l}\text { Qualité, } \\
\text { quantité et } \\
\text { délais } \\
\text { Motivations et } \\
\text { aléas }\end{array}$ \\
\hline $\begin{array}{l}\text { Commande : « On a ce système-là, il fonctionne mais il est complexe } \\
\text { (1). C'est un truc qui est compliqué pour un nouveau boulanger. Déjà } \\
\text { ce qui est compliqué c'est de lui dire la fiche de fabrication tu la fais } \\
\text { vendredi mais pour une fabrication lundi et des livraisons mardi (2). } \\
\text { Il faut avoir un cerveau assez bien fait pour comprendre, moi je ne } \\
\text { comprenais rien au départ. [...] Nous aujourd'hui on se rend compte } \\
\text { que c'est une difficulté pour les boulangers parce que c'est un sacré } \\
\text { boulot d'aller récupérer les mails, déjà se rendre compte si les clients } \\
\text { n'ont pas oublié d'envoyer le mail » }\end{array}$ & $\begin{array}{l}\text { Règles de } \\
\text { fonctionnement } \\
(2) \\
\text { Ressources } \\
\text { humaines } \\
\text { Outils de } \\
\text { gestion }\end{array}$ & $\begin{array}{l}\text { Règles de } \\
\text { fonctionnement } \\
\text { (1) } \\
\text { Flux } \\
d^{\prime} \text { information }\end{array}$ \\
\hline $\begin{array}{l}\text { Elaboration des menus : « Alors déjà on a des outils informatiques } \\
\text { pour la gestion des stocks et les prix de revient, donc on suit au jour } \\
\text { le jour les stocks et les inventaires, qui nous permettent de dire } \\
\text { combien on a dépensé par repas [...] Sur la partie nutritionnelle, on a } \\
\text { une commission menu qui valide les menus, on les fait aussi valider } \\
\text { par une diététicienne à l'extérieur, et on les met en ouvre dans ces } \\
\text { critères qui sont prévus par la loi. Vous avez en particulier le } \\
\text { référentiel du GRCF qui nous fixe quelques recommandations ou } \\
\text { obligations, et sur lequel on reste évidemment en lien » }\end{array}$ & $\begin{array}{l}\text { Outils de } \\
\text { gestion } \\
\text { Règles de } \\
\text { fonctionnement } \\
\text { Pilotage } \\
\text { Normes restau } \\
\text { collective }\end{array}$ & \\
\hline
\end{tabular}




\subsection{Une première compréhension du travail des acteurs qui participent aux processus de reterritorialisation des systèmes alimentaires}

D'abord nous illustrons les deux natures de coordination, leurs relations et leur coévolution, en exposant le cas de la ferme A. Puis nous évoquons l'hétérogénéité des processus de coordination inter-organisationnelle et leur hybridation en présentant le cas de la ferme B. Enfin nous caractérisons un travail de reterritorialisation en mobilisant le cas de l'association C. L'ensemble vise à rendre compte de la multiplicité et de la diversité des réseaux d'acteurs qui participent de près ou de loin au développement d'un système alimentaire visant à privilégier l'offre présente sur le plateau de Saclay pour la restauration d'entreprise.

\subsubsection{Une coévolution des processus de coordination interne et externe pour construire un système alimentaire organisé localement}

Un premier résultat est que les acteurs (organisations) doivent régulièrement questionner voire ajuster les processus de coordination interne et externe, afin de coconstruire avec les autres acteurs un système "organisé » d'approvisionnement des lieux de travail en produits locaux. Cette évolution des processus de coordination implique aussi de repenser les activités de travail en interne, celles qui sont intrinsèquement liées à la relation inter-organisationnelle (gestion des contrats clientfournisseur, livraison, etc.), mais aussi celles qui concernent plus directement la production et la transformation des denrées. Nous présentons ce mécanisme de coévolution des processus de coordination interne et externe à travers le cas de la ferme A.

La ferme A est une exploitation familiale qui existe depuis 1920. Dotée d'un modèle agricole tourné vers les grandes cultures, la transition vers plus de durabilité de la ferme commence en 2003 lorsque la troisième génération d'agriculteurs décide de participer à la création d'une AMAP pour satisfaire une demande citoyenne. En 2011, l'AMAP demande à être approvisionnée en pain et farine. La ferme crée alors un fournil, ce qui entraine un grand changement de l'activité de travail avec l'apprentissage de la meunerie et de la boulangerie. En 2012, 40 \% des surfaces agricoles de la ferme sont converties à l'agriculture biologique. Depuis l'ouverture du fournil, la demande croissante en produits locaux a permis aux exploitants d'augmenter leur production et de vendre leurs produits dans la boulangerie à la ferme qu'ils ont ouverte. Ils fournissent également des magasins Bio, des écoles et des cantines d'entreprises.

Les activités du fournil impliquent le travail de sept personnes: trois boulangers, un vendeur en boulangerie, un livreur et deux exploitants agricoles. L'un des exploitants s'est formé au métier de boulanger pour pouvoir remplacer ou compléter les trois boulangers, si nécessaire. Le deuxième exploitant gère la partie administrative du fournil : élaboration des contrats avec les clients, gestion des factures et des paiements, gestion des plannings des boulangers et du livreur, etc. Le fournil est ouvert du lundi au vendredi au public, et un samedi par mois pour l'AMAP. Les livraisons se font uniquement du lundi au vendredi. 
La figure 1 illustre l'étape du référencement lorsqu'un restaurant d'entreprise souhaite ajouter la ferme A parmi ses fournisseurs. Pour la ferme A, devenir le fournisseur d'un nouveau client peut impliquer une réorganisation plus ou moins importante des activités du fournil. Par exemple, il faut évaluer voire ajuster les productions journalières et hebdomadaires des boulangers. Une nouvelle collaboration avec un restaurant peut également impliquer l'achat de matériel supplémentaire (camion de livraison), l'embauche et la formation de nouveaux salariés (boulanger, livreur). Ainsi, les négociations qui s'opèrent traditionnellement entre un client et un fournisseur sur le prix du produit, la qualité et la quantité souhaitées, ou encore la durée de collaboration pressentie - sont ici sous-tendues par la possibilité qu'a le fournil de se réorganiser pour répondre à la demande. Les critères qui déterminent la collaboration concernent certes la qualité des produits, la quantité et les délais attendus, mais aussi les ressources humaines et matérielles en interne.

La réorganisation du fournil concerne aussi bien les activités qui ont trait à l'approvisionnement des restaurants, que celles qui ont trait à la vente en boulangerie qui reste le client privilégié. En filigrane, c'est la capacité de production qui est interrogée au regard de la satisfaction des clients de la boulangerie et du bien-être des salariés du fournil. Ainsi les exploitants agricoles disent avoir déjà refusé certains clients dont la demande de production allait mettre en péril les horaires de travail des boulangers et du livreur, ou bien allait réduire la quantité produite pour la boulangerie. Les exploitants ont à cœur de respecter certains principes qui garantissent, selon eux, la qualité de vie au travail. D'ailleurs la mise en place d'une collaboration avec un nouveau client est une démarche participative entre les gérants et les salariés, ce qui permet de trouver une organisation de travail qui convienne à tout le monde. Ainsi, deux autres critères déterminent la mise en place d'une collaboration clientfournisseur : le respect d'un pilotage distribué entre les exploitants et leurs salariés, et le respect de règles internes de fonctionnement qui garantissent un travail décent.

«On a pris un troisième boulanger, justement pour garder l'équilibre de nos contrats et de leur qualité de vie. C'est très important pour nous de faire travailler $\mathrm{du}$ lundi au vendredi, pas le samedi, sauf ceux de l'AMAP [...] on ne veut pas travailler la nuit, on ne veut pas qu'eux travaillent la nuit, et tout ça, ça fait qu'il y a une quantité et on essaie de garder cet équilibre [...] On le décide avec nos boulangers. On leur dit qu'il y a un nouveau client qui voudrait tant, qu'est-ce que vous en pensez, est-ce qu'on peut le faire et éventuellement quel jour? S'ils disent on ne peut pas le faire, alors c'est non » (Ferme A). 
Figure 1 : Mise en place d'une collaboration entre la ferme $A$ et un restaurant : éléments déterminants dans le cas du référencement.

Figure 1: implementation of a collaboration between farm $A$ and a canteen : decisive elements regarding referencing

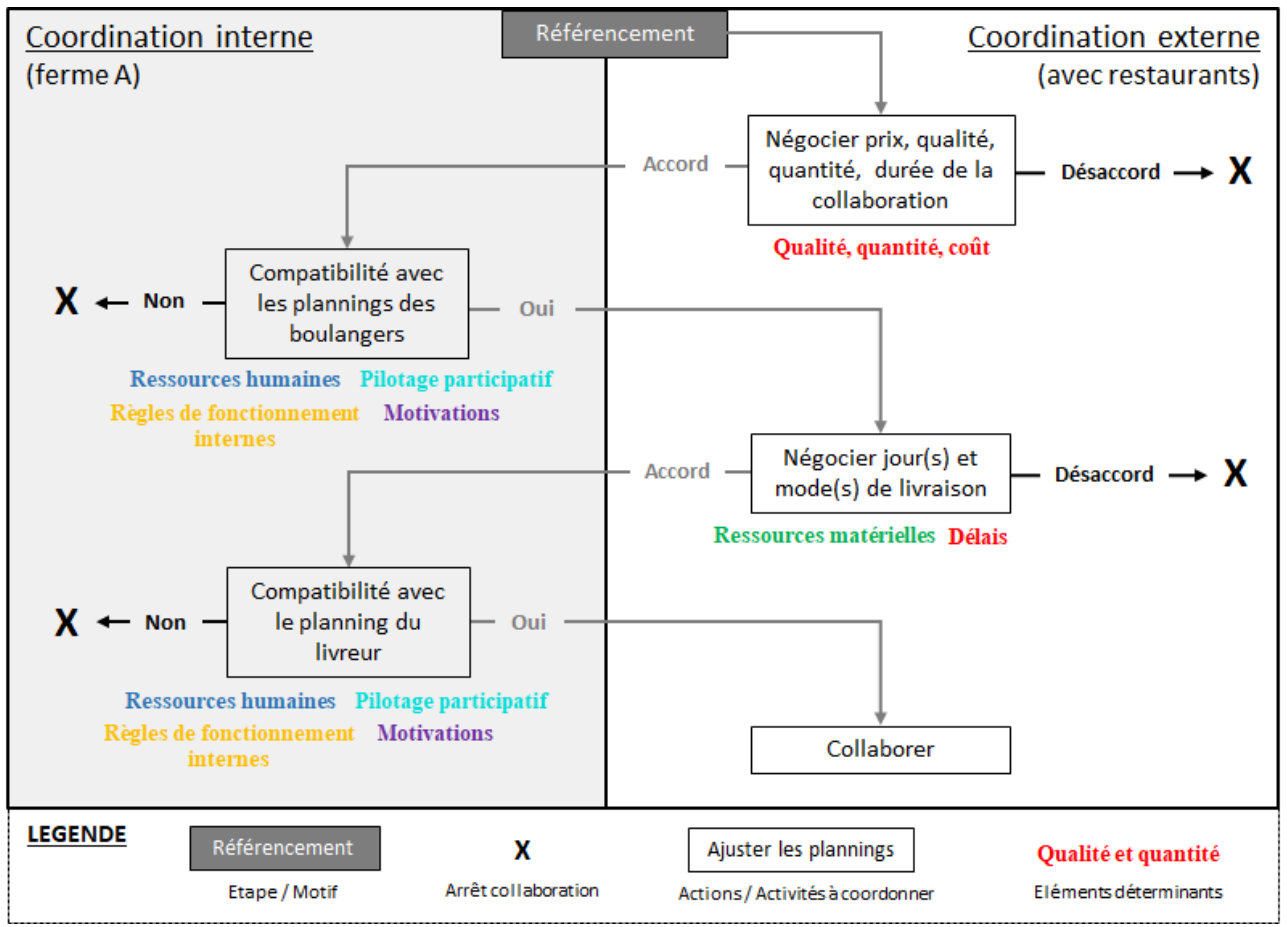

Le respect d'une certaine organisation de travail est aussi perçu par les exploitants comme le gage de pérenniser un procédé de fabrication artisanale. En effet, il s'agit d'un pain au levain fait entièrement à la main, avec un temps de fabrication très long : "C'est nous qui établissons les quantités parce qu'au-delà de certaines quantités nous ne pouvons plus faire. C'est vraiment un travail artisanal qui demande un certain nombre d'heures pour avoir un produit final et donc si on voulait augmenter la quantité il faudrait revoir toute la chaîne de production, et nous ne voulons pas " (Ferme A). L'automatisation de la production n'est pas une solution envisagée, car elle diminuerait la qualité des produits au regard des critères que retiennent les exploitants agricoles pour définir cette qualité. Ils font plutôt le choix d'adapter le nombre de clients à la capacité de production de leur fournil. Ainsi, la mise en place d'une collaboration client-fournisseur repose également sur la motivation de conserver des méthodes de travail qui garantissent un produit « fait-main ».

Par ailleurs, le fournil fonctionne en flux «tirés », c'est-à-dire que la fabrication des produits est lancée uniquement sur précommande afin d'éviter les surstocks et le gaspillage. Ainsi les clients doivent passer leur commande au plus tard l'avant-veille avant $11 \mathrm{~h}$ du matin. Les commandes sont réceptionnées par les boulangers qui mettent à jour l'outil de recensement des commandes clients dans un tableur Excel développé par l'exploitant. Ce mode de fonctionnement est perçu par les exploitants comme compliqué à gérer, d'autant plus que chaque client a ses propres règles de fonctionnement : certains clients établissent un bon de commande pour plusieurs jours, d'autres pour un mois complet, etc. Une autre variabilité importante concerne les périodes de moindre affluence dans les restaurants (par exemple, les vacances d'été) et les périodes de fermeture des restaurants (par exemple, les jours fériés). 
« Eux ils envoient un programme pour la semaine, le vendredi. Lui il l'envoie dans la nuit de dimanche à lundi, c'est un truc qui est fait automatiquement. Sauf que quand il n'y a personne pour s'occuper du truc et bah il n'y a pas de commande. [...] Nous on réserve ce temps-là. Tu vois la difficulté ? [...] Pendant les vacances la production ça s'arrête, donc la consommation » (Ferme A).

Le mode de gestion des commandes en interne entraine aussi une complexification du travail des boulangers les conduisant à mener des tâches qui s'éloignent de celles de leur métier traditionnel. D'abord, ces derniers manipulent des outils de gestion (tableau des clients, comptabilisation des commandes, etc.), dont l'appropriation peutêtre assez coûteuse. Ensuite, les boulangers doivent parfois résoudre des aléas liés à la réception des commandes. Il peut s'agir de commandes erronées, non conformes à ce qui a été conclu au début de la collaboration, d'oublis de commandes, de commandes passées en retard, etc.

« Nous aujourd'hui on se rend compte que c'est une difficulté pour les boulangers parce que c'est un sacré boulot d'aller récupérer les mails, déjà se rendre compte si les clients n'ont pas oublié d'envoyer le mail, parce qu'ils oublient de t'envoyer le mail, mais par contre quand le livreur ne passe pas ils t'appellent! Donc avant ils faisaient la commande systématiquement, et je leur ai dit non » (Ferme A).

La facturation des commandes et la réception des paiements sont gérées par un des deux exploitants. Ce dernier doit s'adapter aux procédures et aux temporalités propres à chaque client. Il doit également s'adapter, parfois, à des clients qui ne sont pas initialement formés à la gestion de fournisseurs (par exemple, des associations étudiantes qui lui commandent du pain). Les exploitants agricoles soulignent certaines contraintes liées à des transactions « de la main à la main », pour lesquelles des erreurs de paiement se produisent fréquemment : « tu leur donnes une facture ils te donnent un chèque qui ne correspond à rien, ni hors taxe, ni TTC, c'est un peu compliqué " (Ferme A). La gestion administrative du fournil occupe donc une place importante parmi l'ensemble des activités des agriculteurs. Ces derniers évoquent une "explosion" des activités de comptabilité et de gestion des informations. Ils se retrouvent avec des périodes où leur charge de travail pour cette activité est conséquente.

«Le $30 \mathrm{du}$ mois arrive toujours quand tu as un milliard de choses autres à faire dans les champs, et par contre tu dois être là parce que tu dois faire les factures. [...] Ce matin j'ai essayé de traiter les mails que tout le monde t'envoie, ils ne sont pas traités depuis 10 jours. Et je n'ai pas réussi à le faire. [...] Au début je mettais les formes sur les mails, maintenant j'envoie la photocopie avec merci de régler, vous devez tant. Sinon t'y passes un temps fou » (Ferme A).

Ainsi l'organisation des activités du fournil et de la ferme dépend fortement de la manière dont les producteurs arrivent à co-construire avec leurs clients une relation basée sur la confiance et l'engagement des différentes parties prenantes. Le nombre de débouchés est relativement limité par la capacité de production du fournil, ce qui permet aux exploitants d'être proches de leurs clients. Ils peuvent avoir des contacts directs et réguliers avec des acteurs jouant des rôles clés dans la distribution des produits locaux, par exemple des responsables de restauration. Ils peuvent également faire un travail de sensibilisation sur les bienfaits de la consommation Bio et locale, via des visites à la ferme. Les relations qui se tissent sont néanmoins fragiles parce qu'elles reposent en premier lieu sur la volonté de chacun des acteurs de poursuivre la collaboration. Lorsque la ferme A devient le fournisseur d'un restaurant, rien ne garantit que ce dernier commandera chez elle de manière régulière et pérenne. Cette 
incertitude est contraignante pour l'organisation du fournil, car la production en flux tirés est un modèle qui est réputé fonctionner dans des contextes de demande relativement stables. De même, l'absence de standardisation des processus administratifs en interne est source d'erreurs et suscite une forme de polyvalence pouvant créer de l'inconfort pour les salariés et les gérants.

Finalement, la ferme A cherche à préserver au mieux ses engagements en matière de développement durable (alimentation de qualité, travail décent) et ses règles de fonctionnement (artisanat), bien qu'elle accepte de faire évoluer ses règles dans le cas où d'autres acteurs partagent ses engagements. Elle conçoit son réseau d'acteurs dans une logique de développement d'un système local éloigné des standards de la restauration collective. Et le territoire qu'elle approvisionne est celui qui prend forme à travers les interactions qu'elle co-construit, autour de son produit alimentaire, avec les acteurs qui partagent ses valeurs en matière d'alimentation locale. Géographiquement, ce réseau est assez proche du Projet Alimentaire Territorial (PAT) dont fait partie le plateau de Saclay.

\subsubsection{Une hybridation des formes de coordinations inter-organisationnelles qui élargit les frontières du système organisé localement}

37 Un deuxième résultat est que les acteurs (organisations) peuvent évoluer au sein de plusieurs réseaux de distribution de produits considérés comme locaux par les acteurs qui in fine les mettent à disposition des consommateurs. De fait, le critère de localité est souvent défini par une distance géographique acceptable plus ou moins grande entre le producteur et le consommateur. Ainsi certains acteurs valorisent cette diversité d'acception du local: ils cherchent d'un côté à contribuer au réseau organisé localement et collaborent avec les acteurs qui défendent un projet alimentaire local et/ ou territorialisé ; et d'un autre côté ils sont insérés dans des réseaux de la restauration collective plus larges en termes de nombre d'acteurs et de répartition géographique des acteurs impliqués. C'est ainsi qu'œuvre la ferme B.

La ferme B est une exploitation laitière qui a débuté ses activités en 1970. Elle a également une activité de maraîchage, et dans les années 80 elle commence à participer à la promotion d'activités agro-touristiques via un regroupement national d'agriculteurs ayant des pratiques agricoles vertueuses et qui proposent des cueillettes à la ferme. Dans les années 90, elle développe des partenariats avec d'autres fermes de l'Ile-de-France pour transformer une partie de son lait en yaourts, faisselles et fromages frais. Les produits sont alors vendus dans la boutique de la ferme. Suite à l'effondrement des prix du lait en Europe en 2012, conduisant à la fermeture de la coopérative qui fabriquait ses yaourts, la ferme B décide d'investir pour rapatrier les activités de transformation laitière dans ses locaux. Cette décision s'est inscrite dans un contexte de forte demande de produits durables et locaux, notamment en restauration collective. En 2018, les produits de la ferme B sont labellisés «produits en Île-de-France ».

Les activités de transformation laitière impliquent le travail d'au moins 15 personnes, dont un responsable commercial qui élabore la stratégie de distribution des produits, un responsable de la logistique, un responsable de la qualité, des agents de production et des livreurs. Les produits sont vendus aux particuliers via la boutique de la ferme et des épiceries fines parisiennes. Les autres clients de la laiterie sont la restauration collective, des boulangers, des fromagers, des restaurants parisiens et des glaciers. La 
figure 2 illustre les deux formes de relation que la ferme B entretient avec trois restaurants d'entreprise du plateau de Saclay que nous avons interrogés : le CROUS de Versailles, l'association C et l'organisme D.

Figure 2 : Formes de relations entre la ferme $B$ et les restaurants.

Figure 2: forms of relationship between farm $B$ and the canteens

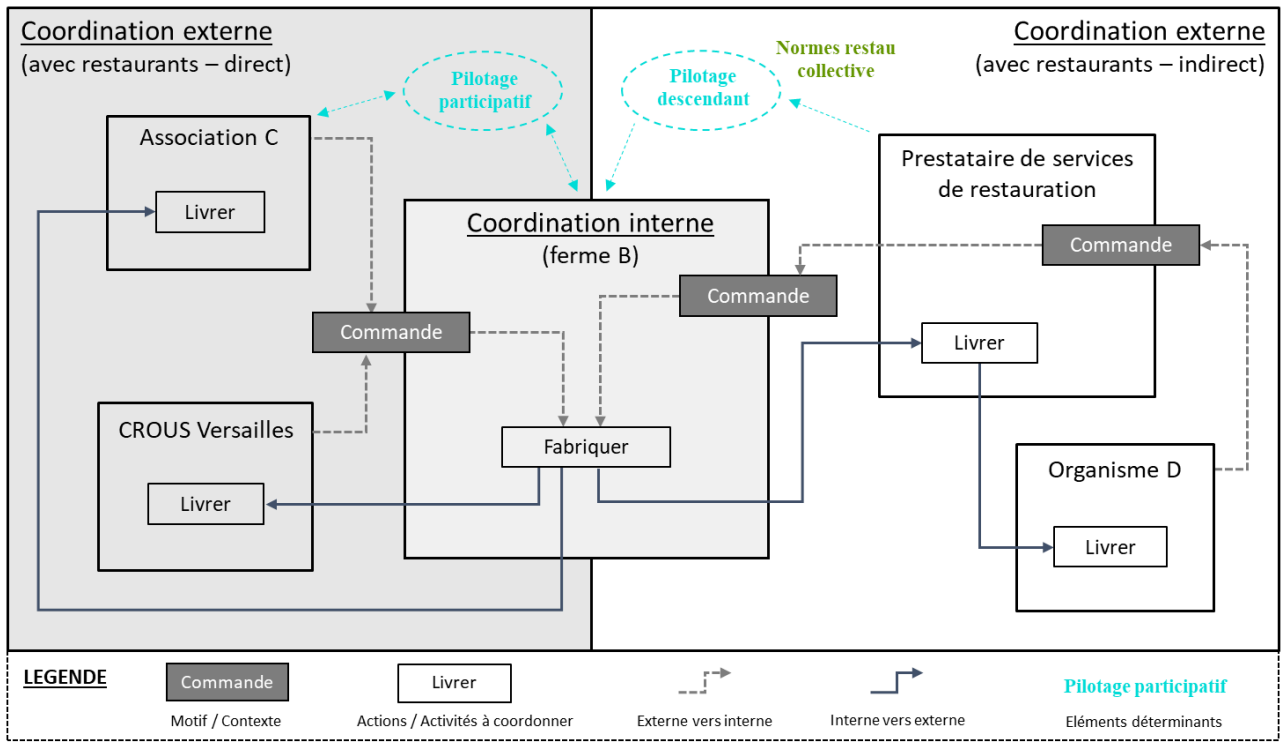

Concernant le CROUS et l'association C, il s'agit d'une relation directe entre la ferme et les restaurants, qui ressemble au modèle présenté dans le cas de la ferme A. Pour le CROUS, la ferme B n'est pas son fournisseur privilégié de yaourts parce qu'il doit fortement veiller à un coût de revient du repas bas étant donné le public à faibles revenus (les étudiants) : "On va dire que la grosse majorité des yaourts vient de l'industrie agroalimentaire, mais on s'en garde une partie aussi avec la filière courte » (un restaurant du CROUS de Versailles). L'association $C$ quant à elle entretient une relation privilégiée avec la ferme $\mathrm{B}$. Elle fut l'un de ses premiers clients et bénéficie à ce titre de tarifs avantageux. En retour, l'association $\mathrm{C}$ n'hésite pas à dépanner la ferme lorsque cette dernière se retrouve avec un surplus de production (légumes, fruits) qu'elle n'arrive pas à vendre. Ainsi cette relation peut prendre la forme d'un pilotage accommodant et participatif. La coordination inter-organisationnelle entre la ferme B et l'association $\mathrm{C}$ repose sur des décisions consensuelles et des règles plutôt souples afin de satisfaire au mieux les deux parties prenantes, et de répondre aux critères de durabilité qui les relient.

« On est un peu privilégié entre guillemets vis-à-vis d'autres clients [...] parce qu'on a travaillé très rapidement avec lui, on commence un petit peu à travailler aussi sur un certain nombre de produits, les légumes, quand parfois il ne trouve pas de débouchés. Il nous a interpellés une ou deux fois sur du chou où il avait une production importante et il n'avait pas de débouchés, il nous a dit: est-ce que ça vous intéresse, on a dit oui. Donc on a changé nos menus et on a pris ses produits qu'il fallait consommer" (Association C qui évoque sa collaboration avec la ferme B).

Au sein de l'organisme $\mathrm{D}$, les restaurants sont concédés à des sociétés de prestation de services de restauration. Les gérants des restaurants sont des employés du prestataire et ils commandent les denrées souhaitées via la centrale d'achats de ce prestataire. Ainsi l'organisme $\mathrm{D}$ a demandé à ses prestataires de référencer les yaourts de la ferme $\mathrm{B}$ 
pour les proposer à la vente dans ses restaurants. Ces prestataires constituent donc des intermédiaires entre le producteur et les restaurants qui ne traitent pas en direct. Les gérants des restaurants alternent les commandes chez différents fournisseurs, dont la ferme B, dans le but de garantir aux convives une offre variée, mais aussi pour équilibrer leurs comptes, car les yaourts de la ferme B sont plus chers que les autres yaourts référencés par la centrale d'achats :

"Le gérant il fait des commandes pour amener de la variété, et il attend que le stock soit terminé avant de commander autre chose. Une fois qu'il a fini ça [yaourt non Bio] il prend des Ferme B, quand il n'y a plus de Ferme B il reprend ça. Comme ça les gens, ça leur fait une variété [...] et puis bon ils sont un peu plus chers hein » (Organisme D).

Les deux formes de relation qu'entretient la ferme B avec la restauration collective sont en fait sous-tendues par une même logique de durabilité qui diffère par certains aspects de celle de la ferme A. Comme elle, la ferme B participe au dynamisme local de distribution de produits locaux en restauration collective et pour les particuliers. Mais elle tisse également des liens d'entraide avec certains responsables de restaurant (association C). Elle vend également dans son épicerie des produits des autres producteurs locaux. Elle mutualise du matériel agricole avec d'autres producteurs. Elle a participé au projet de construction de la Zone de Protection Naturelle Agricole et Forestière (ZPNAF). Ainsi ses activités s'inscrivent dans le système qui est organisé localement par les autres acteurs (producteurs, restaurateurs, société civile) pour contribuer à défendre ce système et ses valeurs, et pour éventuellement l'adapter à ses propres besoins et caractéristiques.

Ensuite, la ferme B est inscrite dans un autre réseau d'acteurs qui lui permet d'accroître ses activités au-delà de ce réseau local. Dans le réseau de la restauration collective concédée, les critères retenus sont principalement ceux de la loi EGalim, laquelle valorise d'abord des critères de qualité et de durabilité, et dans une moindre mesure le critère de la localité. Contrairement à la ferme $\mathrm{A}$, la ferme $\mathrm{B}$ accepte de se conformer aux exigences de la restauration collective. Elle accepte aussi de se conformer aux exigences des consommateurs. Ainsi la relation qu'elle entretient avec les sociétés de restauration sont sous-tendues par les normes dominantes de la restauration collective. Par exemple, la démarche «Bleu-Blanc-Cœur» $(\mathrm{BBC})^{8}$, que la ferme B a initiée, lui permet de faire rentrer ses yaourts dans la case des produits à Haute Valeur Environnementale reconnus par la loi EGalim. De même, la ferme B a récemment modifié les valeurs nutritionnelles de sa recette traditionnelle de yaourt en diminuant les quantités de sucre et de confiture, d'une part pour répondre à des remontées de ses clients et des convives; et d'autre part pour s'aligner sur les recommandations du Programme National Nutrition Santé (PNNS) que les cantines sont tenues de respecter.

La ferme B repense donc ses processus administratifs ainsi que ses activités de production et de distribution de sorte à pouvoir pleinement se coordonner avec les enseignes régionales et nationales de la restauration collective. Les coordinations interorganisationnelles que la ferme construit au sein d'un réseau plus élargi que son réseau local s'inscrivent dans une démarche qui vise à atteindre les critères de durabilité qu'elle souhaite revendiquer. Les évolutions technologiques, organisationnelles et de procédés qu'elle développe lui permettent d'opérer sa transition vers toujours plus durabilité telle qu'elle se dessine en particulier à travers la loi EGalim. Cependant le critère de la localité reste une de ses priorités, bien que ce critère fasse référence à un territoire plus large que celui du PAT de Paris-Saclay. En effet la ferme B ne souhaite 
pas que ses produits soient vendus au-delà de l'Ile-de-France. À cet égard, son réseau d'acteurs est majoritairement francilien: les autres fermes du plateau, l'association locale qui défend la préservation des terres agricoles sur le territoire, l'organisme francilien qui a soutenu le développement du label « lait d'Île-de-France », etc.

\subsubsection{Un travail qui transcende les frontières du territoire pour permettre au système organisé localement de se développer}

Un troisième résultat est que les acteurs (organisations) de l'offre et de la demande des produits locaux en restauration collective peuvent mener un travail qui transcende les frontières du territoire Paris-Saclay dans le but de développer l'offre d'une alimentation durable dans les restaurants du territoire, et en restauration collective plus généralement. Ce travail s'appréhende comme l'ensemble des activités déployées auprès des acteurs publics et des institutions - ou visant à impliquer ces acteurs - pour modifier le fonctionnement dominant et/ou pour produire des nouvelles idées et des nouvelles règles favorables à la valorisation des productions locales. Nous présentons le cas de l'association $C$ qui, d'une part participe au développement du projet alimentaire local sur le territoire Paris-Saclay, avec les contraintes qui lui sont imposées et les ressources (humaines, matérielles) dont elle dispose ; d'autre part est insérée dans des réseaux d'acteurs qui œuvrent pour l'accroissement de ces ressources. « $C$ » est une association loi 1901 dont l'une des missions est la gestion de la restauration du personnel d'un établissement public. Elle comprend un bureau d'élus (président, viceprésidents, secrétaire, trésoriers), du personnel de droit privé et des fonctionnaires. L'association gère trois restaurants et une sandwicherie. Chaque restaurant possède sa propre cuisine et comptabilise entre 16 et 20 employés : un chef de cuisine, un second de cuisine, des cuisiniers, un magasinier, des agents de restauration et des plongeurs. Les restaurants sont ouverts le midi, du lundi au vendredi, et environ 230 jours par an. Chaque année, le responsable de la restauration fait valider par les élus un projet financier prévisionnel pour le fonctionnement des restaurants (nourriture, matériel, main-d'œuvre). Ce responsable s'occupe « de fixer les règles du jeu », que ce soit sur le thème de l'hygiène, de la nutrition et de l'offre de repas. Il fait des propositions aux élus qui les valident ou non.

La figure 3 schématise les réseaux d'acteurs pour la partie restauration de l'association C. Trois échelles de coordination sont illustrées : 1) les coordinations à l'échelle des restaurants (interne) ; 2) les coordinations inter-organisationnelles qui s'opèrent avec les producteurs locaux (externe); et 3) les coordinations qui se réalisent à une échelle plus macroscopique (institutions, politiques publiques, associations et sociétés nationales). La distinction de plusieurs échelles renvoie ici à la complexité des réseaux d'acteurs au sein de chaque échelle, en termes de règles de fonctionnement et de capacité dont disposent les acteurs pour faire évoluer ces règles et les coordinations (structure sociale stabilisée dans le temps vs. structure qui peut évoluer dans un temps plus court). Ainsi une échelle "supérieure " est considérée comme plus complexe et plus stable qu'une échelle "inférieure ", dans le contexte ici de l'approvisionnement de la restauration collective d'entreprise en produits locaux.

À l'échelle intra-organisationnelle (coordination interne), la figure 3 schématise de manière succincte le processus par lequel l'association $C$ fournit des repas aux salariés. D'abord, les menus sont élaborés par une commission qui regroupe le responsable de la restauration, le personnel administratif, les élus, une diététicienne, des cuisiniers et des 
représentants des convives. La commission valide les menus pour une vingtaine de jours, ce qui correspond généralement aux règles appliquées pour le respect de l'équilibre alimentaire de repas proposés. L'élaboration des menus repose sur l'expérience des acteurs en termes de prix et de qualité des denrées. Ainsi, l'équipe de restauration a mis en place des outils de gestion qui lui permettent de tenir des inventaires et de comptabiliser précisément combien a été dépensé à chaque repas. Cette comptabilité précise est importante pour l'approvisionnement en produits locaux (souvent plus chers) parce qu'elle permet d'identifier les ajustements possibles.

«On rentre tous les produits que l'on reçoit dans un logiciel pour pouvoir les ressortir après, les quantifier, les valoriser et identifier le coût de notre repas. Quand on reçoit un produit, on met systématiquement l'origine des produits et le signe de qualité. À chaque moment nous pouvons dire le pourcentage de produits que nous avons par rapport à leur origine ou leur signe de qualité [...]. Et puis si c'est trop haut on applique des modifications. Soit on vérifie ce qui a été consommé, pourquoi ça a été consommé, est-ce que les grammages ne sont pas bons, est-ce que le prix est trop élevé, est-ce qu'il y a eu trop de sorties, est-ce que le repas coûte plus cher que prévu [...] à partir de ces conclusions on prend des mesures pour revenir au prix de revient (visé) » (Association C).

Figure 3 : Réseaux d'acteurs de l'association $\mathrm{C}$ pour ses activités de restauration et éléments déterminants pour assurer le fonctionnement des coordinations internes et externes.

Figure 3: network of stakeholders of association C concerning its catering activities and decisive elements ensuring the functioning of both internal and external coordination

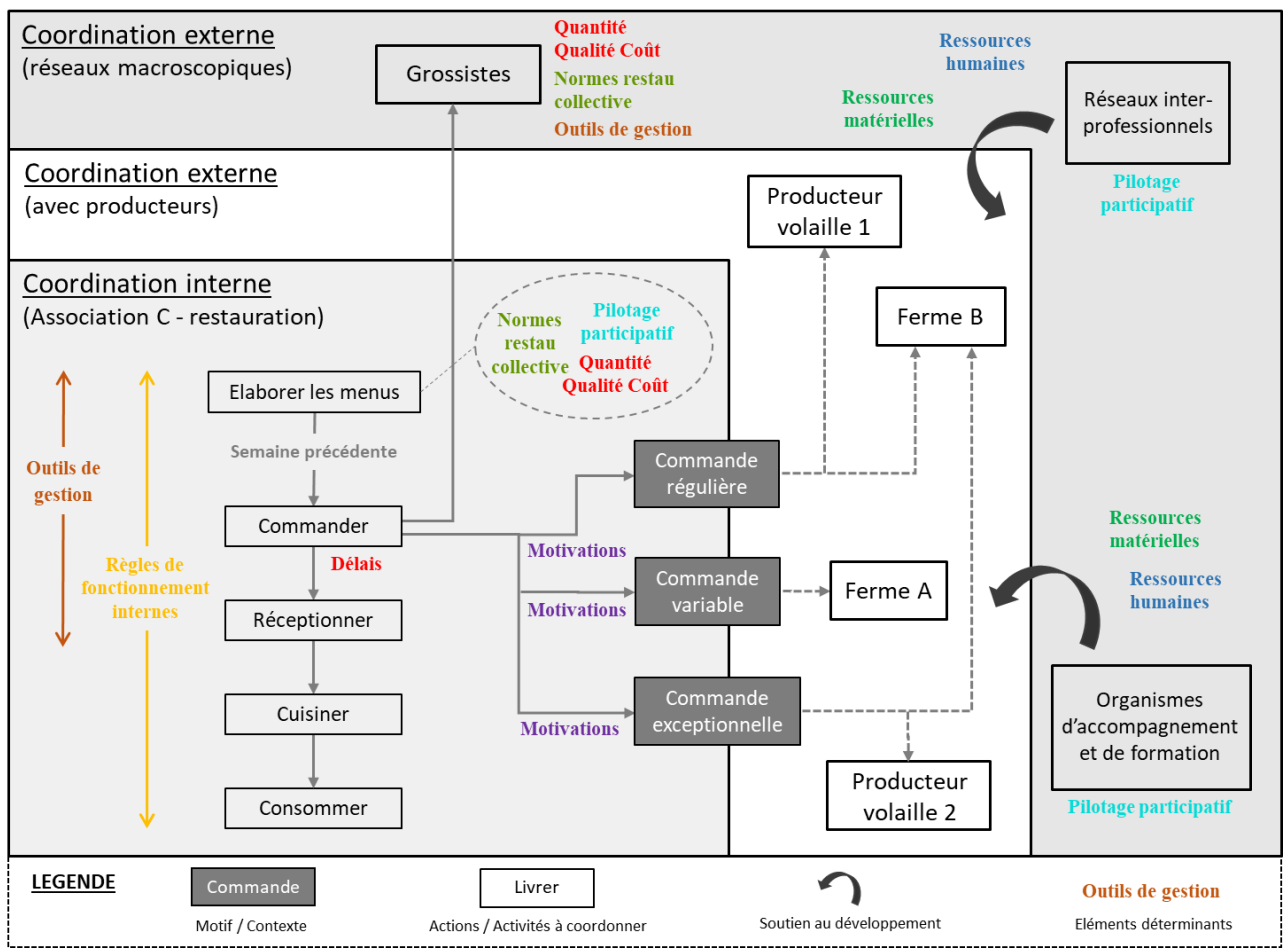

Une fois que les menus sont élaborés, chaque restaurant formule une semaine à l'avance une "expression de besoins" auprès de l'acheteur de l'association, qui centralise les demandes et passe les commandes auprès des fournisseurs. Les produits sont ensuite livrés directement dans les restaurants concernés suivant un agenda de livraison établi avec le fournisseur. Environ trente fournisseurs sont référencés. Ils sont sélectionnés pour un an, renouvelables deux fois, sur la base d'un cahier des charges et de critères majoritairement en lien avec la qualité, le prix et l'origine des produits. La 
confection des repas repose quant à elle sur des fiches techniques qui définissent la recette et qui sont créées régulièrement par les cuisiniers.

La réflexion sur les produits locaux a été initiée il y a une dizaine d'années par le responsable de la restauration qui souhaitait développer un peu plus de liens avec les producteurs locaux et accroître l'approvisionnement en produits français. L'objectif principal était d'améliorer la proximité des produits, puis dans une moindre mesure de développer des signes de qualité. Pour mettre en œuvre ce projet, le responsable de la restauration souligne l'importance de tisser une relation de confiance dès le départ: "Si c'est juste pour un coup ça intéresse peu. Il faut bâtir quelque chose sur du long terme, d'où la création de confiance, d'où un prix remodifié ou en tous cas au juste prix. Et puis on crée comme ça des liens qui sont plus forts ». À cet égard, les relations qu'entretient l'association $C$ avec les producteurs locaux sont diverses et variées. Elles visent à s'adapter aux caractéristiques et aux besoins des fermes. On peut néanmoins faire ressortir trois catégories de relations :

1. L'approvisionnement régulier, lorsque le producteur livre des volumes préétablis à des temporalités fixes. Sur la figure 3, c'est le cas du producteur de volaille $1:$ « lui, c'est un contrat à l'année, mais livraison mensuelle systématique d'un volume préétabli. Donc ça lui permet d'assurer un complément intéressant pour sa structure » (Association C).

2. L'approvisionnement variable, lorsque les volumes ou les jours de livraison ne sont pas stables dans le temps. Sur la figure 3, c'est le cas de la ferme A : « comme je suis ouvert plus que les cantines scolaires, le différentiel c'est moi qui l'achète pour qu'il puisse payer son personnel, donc j'achète un peu plus de pain quand les écoles sont fermées. Et puis lui il a modéré son prix » (Association C).

3. L'approvisionnement exceptionnel lorsque les restaurants « dépannent » un producteur qui doit trouver un débouché rapidement pour ses produits. Sur la figure 3, c'est le cas de la ferme $B$ qui fournit parfois des légumes en plus de ses produits laitiers de manière régulière. Mais c'est aussi le cas de producteurs qui ne sont habituellement pas dans le réseau d'acteurs des restaurants, tels que le producteur de volaille 2 . Le responsable de la restauration n'hésite pas à repenser les règles qu'il a lui-même prescrites (par exemple, l'élaboration des menus) pour défendre sa vision de l'alimentation locale.

« Notre idée c'est aussi de soutenir l'agriculture. Si on doit changer un menu parce qu'un agriculteur du coin a des produits en trop, on ne va pas gâcher. C'est vrai sur notre territoire et c'est vrai aussi ailleurs. J'ai été interpellé une fois par un volailler qui était sur Rennes et qui avait perdu son contrat et qui avait 500 volailles, mais il ne savait pas quoi en faire. On a réussi à plusieurs à lui prendre tout son stock pour lui permettre d'épuiser sa marchandise, et lui assurer un rebond » (Association C).

Ces trois modalités d'approvisionnement manifestent une volonté de soutenir l'alimentation locale sur le territoire Paris-Saclay, mais aussi au-delà de ses frontières. Par exemple, le producteur de volaille 1 se situe sur le département de l'Essonne, mais dans une ville extérieure au PAT du plateau de Saclay. L'élargissement du critère de localité au-delà du territoire est consécutif à l'absence de certains produits sur ce territoire. En d'autres termes, le territoire ne propose pas suffisamment de ressources alimentaires pour répondre aux besoins de la restauration collective locale. À cet égard, on assiste à nouveau à une hybridation des coordinations inter-organisationnelles avec par exemple l'insertion de l'association $C$ au sein de réseaux d'acteurs plus "dominants", tels que les entreprises nationales de distribution de produits alimentaires. Ces dernières peuvent être des ressources pour s'approvisionner «le plus localement possible ». De plus, elles permettent au restaurateur d'avoir accès à des 
produits moins chers et de gérer un seul interlocuteur plutôt que de multiples fournisseurs.

" Je passe par des grossistes parce que je ne vais pas trouver un agriculteur qui va tout me fournir. Par contre, [le grossiste] a regardé autour pour voir s'il y avait des agriculteurs qui travaillaient, et il a trouvé un fournisseur de tomates qu'il a référencé. Et j'ai pu comme ça bénéficier d'un producteur local qui puisse me livrer des tomates plein champs à côté de chez moi » (Association C).

Néanmoins l'association $\mathrm{C}$ ne se contente pas du fonctionnement dominant, mais cherche à développer ses capacités d'action et de changements auprès d'acteurs plus institutionnels, et cela se retrouve ensuite dans les coordinations interorganisationnelles avec les producteurs locaux et dans les situations de travail. Les relations qui s'opèrent avec des réseaux d'acteurs plus macroscopiques visent l'accroissement des ressources matérielles et humaines pour permettre au système alimentaire local de se développer.

Par exemple, l'association C est très active au sein d'un réseau interprofessionnel de la restauration collective qui accompagne les politiques alimentaires auprès des pouvoirs publics. Lors de l'élaboration de la loi EGalim, ce réseau a notamment contribué à l'élévation du seuil à partir duquel les fournisseurs candidats sont mis en concurrence via un appel d'offres. Ce seuil est passé de 25000 euros à 40000 euros hors-taxe par an, favorisant ainsi un achat de "gré à gré " plus conséquent entre les producteurs locaux et les restaurants. De même, ce réseau œuvre auprès de l'Éducation Nationale pour la création de formations spécifiques aux métiers de la restauration collective, pour une reconnaissance de ces métiers et pour une valorisation des compétences. En effet, la restauration collective implique des savoir-faire communs à l'ensemble des métiers de bouche, mais aussi des savoir-faire spécifiques qui sont d'autant plus nécessaires pour la réintroduction de produits frais et locaux (par exemple, «la découpe d'un poulet entier »). À cet égard, des acteurs tels que des organismes de formation privés entrent en jeu pour permettre la montée en compétences des acteurs concernés.

\section{Quelles perspectives ouvertes pour l'analyse et l'intervention ergonomiques?}

53 Premièrement, notre étude de cas illustre que les valeurs de certains acteurs des Systèmes Alimentaires Territorialisés (SAT) les amènent à élargir la notion de reterritorialisation de l'alimentation en incluant des approvisionnements non locaux, mais porteurs d'attributs qui sont pour eux aussi importants à promouvoir. Ainsi, elle met en avant l'intérêt, dans l'étude des SAT, de ne pas s'intéresser qu'à des initiatives à fort ancrage territorial ou local, mais aussi à la façon dont une diversité d'organisations se reconfigure (en interne et dans leurs relations avec l'extérieur) pour intégrer des critères de durabilité et de localité dans une logique de performance autant que de reterritorialisation de leur offre en alimentation.

Deuxièmement, les organisations qui ont été présentées illustrent la propagation de contraintes à l'origine de la transformation des pratiques de coordination intra et inter-organisations. Cette transformation est inhérente au développement d'une alimentation locale au sein de la restauration au travail sur le territoire Paris-Saclay. Notre grille d'analyse, inspirée par les approches gestionnaires, pointe la manière dont les acteurs font évoluer des éléments de coordination intra et inter-organisationnels 
(règles, outils, délais, procédures, etc.) dans le but de privilégier un lien de proximité géographique et de développer plus globalement une offre en alimentation durable et de qualité au sein de la restauration collective. De manière analogue, notre rapprochement avec les sciences de gestion nous a permis d'intégrer la question du modèle économique que les organisations souhaitent préserver ou atteindre, en lien avec les valeurs de durabilité qu'elles recherchent, et les coordinations qu'elles reconçoivent en interne et en externe pour articuler ces deux enjeux. Ainsi, la proximité géographique ne s'exprime pas uniquement dans la distance parcourue entre la production et la consommation. Elle s'exprime aussi dans les arrangements organisationnels et inter-organisationnels qui visent à soutenir les critères de durabilité économique et sociale des acteurs (modèle économique viable pour les parties prenantes, travail décent, travail artisanal), parmi lesquels se retrouve le critère de localité.

De notre point de vue, notre étude de cas s'inscrit pleinement dans les travaux en ergonomie qui s'attachent à traiter d'activités qui dépassent les frontières de l'entreprise (Bationo-Tillon, Poret, \& Folcher, 2020). Elle s'inscrit aussi dans les travaux qui réinterrogent à un niveau " macro ", les marges de manœuvre et les capacités à agir des acteurs opérant à un niveau plus "micro» (Boudra et al., 2019; Cunha, \& Lacomblez, 2009). Il s'agit de rendre compte de l'enchevêtrement de systèmes d'acteurs (le collectif de travail, l'entreprise, la communauté locale ou encore la chaîne de valeur) et d'interroger les répercussions qu'ont les enjeux sociétaux (par exemple, le développement durable) sur les activités et les situations de travail (Hasle, \& Jensen, 2012; Thatcher, \& Yeow, 2016). Dans ces approches multi-scalaires, les systèmes d'acteurs ont été appréhendés sous l'angle du pouvoir décisionnaire et de l'objet du travail. Par exemple, Boudra et al. (2019) montrent que la prévention des risques professionnels dans un contexte de territorialisation d'une fonction sociétale comme le tri des déchets nécessite un arbitrage entre trois niveaux décisionnels. Le niveau « macro » est celui des politiques nationales qui définissent le cadre de l'action avec des directives globales incitatives ou coercitives. Le niveau «méso » est celui des acteurs qui se coordonnent pour mettre en œuvre ces directives à l'échelle territoriale. Le niveau «micro» est celui de l'entreprise dans laquelle l'activité de tri des déchets s'exécute et où la prévention s'applique.

Notre étude de cas éclaire les coordinations qui se mettent en place entre les acteurs pour une offre en alimentation locale sur les lieux de travail, et pour construire ou reconfigurer un système alimentaire territorialisé. Elle nous a permis dans un premier temps de mettre en lumière la dynamique de ces coordinations à des niveaux intra et inter-organisationnels, et de pointer le travail d'organisation (de Terssac, 2003) réalisé par des acteurs qui souhaitent atteindre des objectifs de durabilité, notamment l'objectif d'une alimentation plus locale. Ces acteurs contribuent en effet à modifier la structure formelle de l'organisation et produisent de nouvelles règles. Cette création de règles se fait dans une activité distribuée entre plusieurs acteurs qui prennent des décisions ensemble, ou qui tiennent compte des répercussions de leurs décisions sur les activités des autres acteurs, et qui mettent en place des actions réversibles pour que le système tout entier fonctionne et que leurs objectifs soient atteints comme l'évoque de Terssac (2009). L'étude ergonomique de cette activité de création de règles par les acteurs eux-mêmes pourrait être approfondie, en mobilisant la grille que nous avons construite à l'interface entre sciences de gestion et ergonomie, mais en la mobilisant pour appréhender cette activité comme prenant place dans un processus de re- 
conception progressive de l'organisation dans l'usage (Arnoud, 2013 ; Petit, 2005). Cela permettrait de mieux saisir l'organisation comme un construit dynamique, en évolution permanente, car émergeant de l'action des acteurs (Lorino, 2013). Et cela permettrait d'approfondir la façon dont les situations de travail comme les activités en leur sein évoluent et en retour viennent questionner ces règles développées dans les processus de reterritorialisation à l'œuvre pour offrir une alimentation durable et locale.

57 Notre étude nous incite aussi à proposer de nommer travail de territorialisation l'ensemble des activités qui permettent aux acteurs de se lier autour d'une certaine vision du local et du durable, au sein d'un espace géographique, culturel et/ou politique qu'ils identifient comme territoire et qu'ils font évoluer. Ici, le territoire serait donc bien une structure ou un cadre, mais aussi un processus (Cunha, à paraître) territorialisant la relocalisation de l'alimentation via les activités de travail. De même que le travail d'organisation (ou de réorganisation) collectif (de Terssac, 2003) vise la création de nouvelles façons de faire, pour un meilleur agencement organisationnel collectif; le travail de territorialisation (ou de reterritorialisation) vise à créer des marges de manœuvre, des nouvelles façons de se coordonner, voire des nouvelles façons de faire, pour permettre d'offrir dans un espace géographique et administratif une alimentation durable et locale. Une première caractéristique de ce travail de territorialisation est qu'il s'opère à l'échelle des coordinations inter-organisationnelles et qu'il vise à les reconfigurer pour permettre au système alimentaire territorialisé de se développer. Une deuxième caractéristique est qu'il prend différentes formes selon les acteurs, leurs besoins, leurs volontés, leurs contraintes et leurs ressources. Les cas des fermes $\mathrm{A}$ et $\mathrm{B}$, et de l'association $\mathrm{C}$, montrent notamment trois manières de contribuer à la reterritorialisation du système alimentaire.

Les travaux existants sur la reconception organisationnelle en ergonomie concernent majoritairement l'échelle de l'entreprise et peu d'études s'intéressent à ce jour aux dynamiques inter-organisationnelles. Des travaux récents évoquent tout de même la nécessité de "voyager » au-delà des frontières de l'organisation (Bationo-Tillon et al., 2020) pour traiter des sujets sociétaux (par exemple, l'égalité femme-homme, la lutte contre les discriminations et le dérèglement climatique) qui se manifestent dans le travail (Petit, 2020). La frontière est ici entendue comme ce qui délimite « ce qui est déjà institué au sein d'une organisation, d'une communauté, de ce qui n'est pas encore développé » (Bationo-Tillon et al., 2020, p.2). Notre étude offre une illustration de la manière dont des acteurs tentent de prendre en main l'organisation d'un système qui transcende les entreprises. À cet égard, une troisième caractéristique du travail de territorialisation est qu'il transcende les frontières de l'entreprise, dans le but de faire avancer le bien commun, ici le projet de relocalisation de l'alimentation dans le territoire. Ainsi certains acteurs ayant des rôles clés dans les coordinations interorganisationnelles (responsable de restaurant, responsable d'exploitation agricole) deviennent des personnes-frontières (Akkerman, \& Bakker, 2011). Elles franchissent les frontières qui existent entre les différents modes de structuration des approvisionnements en produits alimentaires sur le territoire (par exemple, les circuits courts et les circuits longs) et construisent ou abattent des ponts entre ces modalités d'approvisionnement via la reconception dans l'usage des coordinations interorganisationnelles. 
59 Du point de vue de l'analyse ergonomique, nous proposons d'étudier le changement inter-organisationnel qui vise la reterritorialisation l'alimentation, comme un processus de conception collective (Arnoud, 2013; Barcellini, Van Belleghem, \& Daniellou, 2013 ; Petit, \& Dugué, 2013) qui met en dialogue deux processus. Le premier est le processus de reconception des coordinations intra-organisationnelles pour permettre la réalisation de l'offre alimentaire durable et locale au quotidien. Il se traduit par l'élaboration de nouvelles modalités de fonctionnement et de nouvelles pratiques situées (dans les restaurants, dans les fermes), parfois en rupture avec celles qui prévalaient jusqu'ici. Ce processus se met en œuvre via un travail d'organisation à l'intérieur de l'entreprise. Le second est le processus de reconception des coordinations inter-organisationnelles qui, au niveau de l'ensemble de la chaîne de valeur, vise à réélaborer la structure formelle qui soutient la coordination des activités de production, transformation, distribution et consommation, en vue d'intégrer des produits considérés comme locaux dans les repas. C'est alors le travail de territorialisation, chaque fois singulier, qui assure l'évolution conjointe de ces deux processus évoluent. Ainsi, une quatrième caractéristique du travail de territorialisation est qu'il inclut en son sein un travail de reconception des coordinations intraorganisationnelles, ainsi qu'une reconfiguration des situations et des activités de travail à l'échelle "micro». Par exemple, l'étude de cas a mis en évidence une reconfiguration voire un "bricolage» des outils de gestion et des procédures en interne pour permettre la vente ou l'achat de produits locaux. Néanmoins il convient pour l'analyse d'aborder le point de vue selon lequel « le tout est plus que la somme des parties ». En ce sens, le travail de territorialisation n'est pas l'addition, ni l'articulation stricto sensu des coordinations internes et externes. Mais il est plutôt une forme particulière d'arrangement de ces deux processus en lien avec des déterminants propres au territoire et aux entreprises concernées. Déterminants qui sont liés aux ressources économiques (par exemple, un coût de revient du repas à respecter, un modèle d'affaire à pérenniser) et ressources naturelles (par exemple, l'étendue et les caractéristiques des terres agricoles). Déterminants culturels et idéologiques (par exemple, une certaine vision de la durabilité à défendre, des produits de terroir à valoriser). Déterminants politiques et législatifs (par exemple, la loi EGalim qui s'applique ou non, l'existence d'un PAT ou non). Déterminants enfin liés aux dimensions humaines et techniques (capacités de travail accessibles et compétences mobilisables, mais aussi les expériences vécues, la possibilité ou bien le souhait de développer une certaine polyvalence professionnelle, ou d'investir dans des innovations technologiques).

Dès lors l'intervention ergonomique devrait favoriser des rencontres entre les acteurs qui sont parties prenantes de ce travail de territorialisation, afin que l'ensemble de ces acteurs qui produisent la structure organisationnelle, puisse être parties prenantes des réflexions sur l'organisation (Petit, \& Dugué, 2013) facilitant un développement adapté aux environnements et attendus territoriaux. Il y a donc un enjeu à la mise en place d'espaces de débats sur le travail entre les acteurs-frontières pour leur permettre de confronter leurs pratiques comme leurs limites et d'ainsi prendre conscience des interdépendances de leurs actions respectives dans le but de faire émerger les conditions préalables à une coordination inter-métiers efficace et respectueuse de la santé (Arnoud, 2013 ; Lorino 2009 ; Petit, \& Dugué, 2013 ; Poret et al., 2016). Dans le cas de la relocalisation des systèmes alimentaires dans les territoires, cela implique de développer des méthodes qui permettraient aux parties prenantes de co-construire des 
nouveaux modes d'organisation qui renforceraient l'offre alimentaire durable sur le territoire, et qui garantiraient un travail respectueux de la santé. Par exemple, l'adaptation de méthodes de simulation organisationnelle et de simulation managériale (Van Belleghem, 2018), ou bien l'usage de jeux de rôle visant à soutenir le débat sur des controverses (Le Bail, Détienne, \& Baker, 2018) mériteraient d'être développés. En outre, l'intervention ergonomique pourrait permettre la mise en évidence du dialogue entre les deux processus de coordination intra et inter-organisationnelle, et créer les conditions pour que leur reconception s'opère en tenant compte du travail aux différentes échelles. Par exemple, un tel accompagnement pourrait être envisagé sur la reconception d'outils et d'instruments de gestion, de sorte à ce qu'ils articulent mieux les deux niveaux de coordination et leurs caractéristiques propres.

61 Néanmoins l'intervention ergonomique pourrait se heurter à la difficulté d'identifier précisément les acteurs (individus et organisations) qui souhaiteraient être accompagnés dans la reconfiguration de leurs activités. De même, l'intervention ergonomique pourrait concerner des demandes qui ont trait directement à une partie des activités de la chaîne de valeur et rencontrer des obstacles pour travailler l'articulation de ces activités avec d'autres activités qui ne seraient pas inclues initialement dans la demande. Notre étude ne permet pas de conclure sur ces enjeux, mais elle ouvre des perspectives pour des futures recherches visant à préciser les rôles d'acteurs qui contribuent à prescrire le changement des coordinations interorganisationnelles. Une première proposition est de positionner l'intervention ergonomique au niveau des projets qui font évoluer le territoire-structure, par exemple les projets alimentaires territoriaux (PAT) ou bien les projets d'aménagement urbain. À cet égard, un de nos objectifs sur le plateau de Saclay est de construire avec l'association locale qui porte le PAT, des ateliers participatifs pour permettre aux acteurs d'horizons divers (collectivités territoriales, concepteurs des espaces de restauration collective, etc.) d'envisager collectivement des futurs désirables. Une deuxième proposition est de situer l'intervention ergonomique au niveau des infrastructures matérielles qui sont mises en place pour reterritorialiser les systèmes alimentaires au service d'une alimentation durable, par exemple les ateliers de transformation, les plateformes de stockage et plus largement les plateformes logistiques «locales ». Pour cela, nous souhaitons étudier davantage la manière dont ces infrastructures cristallisent des coordinations inter-organisationnelles, révèlent des logiques économiques et de solidarité, offrent un espace de négociation des conventions sociales, et constituent éventuellement des ressources pour le développement des activités, notamment celles des personnes-frontières.

\section{BIBLIOGRAPHIE}

Akkerman, S., \& Bakker, A. (2011). Boundary Crossing and Boundary Objects. Review of Educational Research, 81(2), 132-169. https://doi.org/10.3102/0034654311404435 
Alphandéry, P., \& Bergues, M. (2004). Territoires en questions : pratiques des lieux, usages d'un mot. Ethnologie française, 34(1), 5-12. https://doi.org/10.3917/ethn.041.0005

Arnoud, J. (2013). Conception organisationnelle : pour des interventions capacitantes. Thèse de doctorat en ergonomie. Conservatoire National des Arts et Métiers de Paris.

Barcellini, F., Van Belleghem, L., \& Daniellou, F. (2013). Les projets de conception comme opportunité de développement des activités. In P. Falzon (Ed.), Ergonomie constructive (pp. 191-206). PUF.

Bationo-Tillon, A., Poret, C., \& Folcher, V. (2020). Appréhender le développement des organisations à la croisée du cours d'action et de l'approche instrumentale : la perspective transitionnelle. Activités, 17(2). https://doi.org/10.4000/activites.5371

Boudra, L., Béguin, P., Delecroix, B., \& Pueyo, V. (2019). Prendre en compte le territoire dans la prévention des risques professionnels. Le cas du travail de tri des emballages ménagers. Le travail humain, 82(2), 99-128. https://doi.org/10.3917/th.822.0099

Cardona, A., Lamine, C., \& Hochereau, F. (2012). Mobilisations et animations autour des réductions d'intrants : stratégies d'intéressement des agriculteurs dans trois territoires franciliens. Revue d'Études en Agriculture et Environnement, 93(1), 49-70. https://doi.org/10.4074/ s1966960712001038

Caroly, S. (2010). L'activité collective et la réélaboration des règles: des enjeux pour la santé au travail. Habilitation à diriger des recherches. Université Victor Segalen Bordeaux 2.

Caroly, S., \& Weill-Fassina, A. (2007). En quoi différentes approches de l'activité collective des relations de services interrogent la pluralité des modèles de l'activité en ergonomie ? Activités, 4(1). https://doi.org/10.4000/activites.1414

Chantre, E., \& Cardona, A. (2014). Trajectories of French field crop farmers moving toward sustainable farming practices: change, learning, and links with the advisory services. Agroecology and Sustainable Food Systems, 38(5), 573-602. https://doi.org/10.1080/21683565.2013.876483

Cunha, L. (à paraître). Les apports de l'ergologie pour une intervention développementale territorialisée. In J. Arnoud, F. Barcellini, M. Cerf, \& M.-S. Perez-Torella (Eds.), Interventions et Développements. Octarès.

Cunha, L., \& Lacomblez M. (2009). L'influence du tracé de la mobilité dans la notion de territoire et dans les opportunités de développement local. Ergologia, 2, 157-186.

Du Tertre, C. (2013). Économie servicielle et travail : contribution théorique au développement « d'une économie de la coopération ». Travailler, 29(1), 29-64. https://doi.org/10.3917/trav. 029.0029

Fabbe-Costes, N., \& Lancini A. (2009). Gestion inter-organisationnelle des connaissances et gestion des chaînes logistiques : enjeux, limites et défis. Management \& Avenir, 24(4), 123-145. https://doi.org/10.3917/mav.024.0123

Flores, S. S., \& Medeiros, R. M. V. (2018). La dimension territoriale du développement durable. Confins. Revue franco-brésilienne de géographie, 38. https://doi.org/10.4000/confins.15992

Geels, F. W., \& Schot, J. (2007). Typology of sociotechnical transition pathways. Research policy, 36(3), 399-417. https://doi.org/10.1016/j.respol.2007.01.003

Guibourdenche, J., \& Cahour, B. (2019). Développement durable : nouvelles perspectives en psychologie ergonomique et ergonomie. Psychologie Française, 64(2), 115-118. https://doi.org/ 10.1016/j.psfr.2019.04.001 
Hasle, P., \& Jensen, P. L. (2012). Ergonomics and sustainability-challenges from global supply chains. Work, 41, 3906-3913. https://doi.org/10.3233/wor-2012-0060-3906

Hatchuel, A., \& Weil, B. (1992). L'expert et le système. Economica.

Hubault, F. (2011). Economie de la fonctionnalité et travail : premiers questionnements. In G. Gaglio, J. Lauriol, \& C. du Tertre (Eds.), L'économie de la fonctionnalité : une voie nouvelle vers un Développement Durable (pp. 85-93). Octarès.

Lamine, C. (2012). « Changer de système » : une analyse des transitions vers l'agriculture biologique à l'échelle des systèmes agri-alimentaires territoriaux. Terrains travaux, 20(1), 139-156. https://doi.org/10.3917/tt.020.0139

Lamine, C., \& Chiffoleau, Y. (2016). Reconnecter agriculture et alimentation dans les territoires : dynamiques et défis. Pour, 232, 225-232. https://doi.org/10.3917/pour.232.0225

Le Bail, C., Détienne, F., \& Baker, M. (2018). A role-playing simulation to support assessment of sustainable sociotechnical systems for and by citizens. In Proceedings of the $36^{\text {th }}$ European Conference on Cognitive Ergonomics. ACM. https://doi.org/10.1145/3232078.3232091

Leplat, J. (1993). Ergonomie et activités collectives. In F. Six, \& X. Vaxevanoglou (Eds.), Les aspects collectifs du travail (pp. 7-27). Octarès.

Livian, F. (1998). Organisation : Théories et pratiques. Dunod.

Lorino, P. (2009). Concevoir l'activité collective conjointe : l'enquête dialogique. Étude de cas sur la sécurité dans l'industrie du bâtiment. Activités, 6(1). https://doi.org/10.4000/activites.2154

Lorino, P. (2013). L'activité collective, processus organisant ; un processus discursif fondé sur le langage pragmatiste des habitudes. Activités, 10(1). https://doi.org/10.4000/activites.656

Marié, M. (2004). L'anthropologue et ses territoires. Ethnologie française, 34(1), 89-96. https:// doi.org/10.3917/ethn.041.0089

Moine, A. (2006). Le territoire comme un système complexe : un concept opératoire pour l'aménagement et la géographie. L'Espace géographique, 35(2), 115-132. https://doi.org/10.3917/eg. 352.0115

Motté, F., \& Haradji, Y. (2010). Construire la relation de service en considérant l'activité humaine dans ses dimensions individuelles et collectives. In G. Vallery, M.-C. Le Port, \& M. Zouinar (Eds.), Ergonomie et conception de produit et de services médiatisés (pp. 11-35). Paris : PUF. https://doi.org/ 10.3917/puf.lepo.2010.01.0011

Muchnik J., Requier-Desjardins D., Sautier D., \& Touzard J. M. (2007). Les systèmes agroalimentaires localisés (SYAL) : introduction. Economies et Sociétés, 29, 1465-1484.

Pesqueux, Y. (2014). De la notion de territoire. Prospective et stratégie, 4-5(1), 55-68. https:// doi.org/10.3917/pstrat.004.0055

Petit, C., Levavasseur, F., \& Verger, Y. (2018). Reconnecter villes et campagnes : vers des symbioses agricoles et agri-urbaines? Une exploration sur le Plateau de Saclay. Vertigo (Horssérie 31). https://doi.org/10.4000/vertigo.21809

Petit, J. (2005). Organiser la continuité du service : Intervention sur l'organisation d'une mutuelle de santé. Thèse de doctorat en ergonomie. Université Bordeaux 2.

Petit, J. (2020). Intervention sur l'organisation : concevoir des dispositifs de régulation pour un travail plus démocratique. Habilitation à diriger des recherches. Université de Bordeaux. 
Petit, J., \& Dugué, B. (2013). Structurer l'organisation pour développer le pouvoir d'agir : le rôle possible de l'intervention en ergonomie. Activités, 10(2). https://doi.org/10.4000/activites.816

Poret, C., Folcher, V., Motté, F., \& Haradji, Y. (2016). Concevoir pour le pouvoir d'agir ensemble au sein des organisations : le cas d'un processus commercial. Activités, 13(2). https://doi.org/ $10.4000 /$ activites. 2820

Porter, M. E. (1998). Clusters and the new economic of competition. Harvard Business Review, 76, 77-90.

Rastoin, J. L., \& Ghersi, G. (2010). Le système alimentaire mondial : concepts et méthodes, analyses et dynamiques. Éditions Quae.

Reynaud, J. D. (1997). Les règles du jeu : l'action collective et la régulation sociale ( $3^{\mathrm{e}}$ édition). Armand Colin.

Terssac de, G. (2003). La théorie de la régulation sociale de Jean-Daniel Reynaud. Débats et prolongements. La Découverte.

Terssac de, G. (2009). Le travail d'organisation : Un objet pour l'ergonomie ? Communication

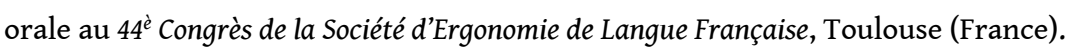

Thatcher, A., Waterson, P., Todd, A., \& Moray, N. (2018). State of Science: ergonomics and global issues. Ergonomics, 61(2), 197-213. https://doi.org/10.1080/00140139.2017.1398845

Thatcher, A., \& Yeow, P. H. P. (2016). A sustainable system-of-systems approach: A new human factors and ergonomics paradigm. Ergonomics, 59(2), 1-12. https://doi.org/ 10.1080/00140139.2015.1066876

Van Belleghem, L. (2018). La simulation de l'activité en conception ergonomique : acquis et perspectives. Activités, 15(1). https://doi.org/10.4000/activites.3129

Yin, R. K. (2003). Case study research: Design and methods (3rd ed.). Sage.

\section{NOTES}

1. Association pour le Maintien d'une Agriculture Paysanne.

2. Nous entendons par là que les objectifs des organisations ne sont pas nécessairement uniquement financiers.

3. Les PAT sont des projets qui œuvrent en faveur de l'autonomie alimentaire locale en structurant et en consolidant des filières alimentaires locales (par exemple, aide à l'installation agricole, aide à la diversification des exploitations vers des débouchés locaux, etc.).

4. Les produits dits durables sont: les produits bénéficiant d'un signe de qualité (Label Rouge, Appellation d'Origine Protégée, Appellation d'Origine Contrôlée, Indication Géographique Protégée) ou d'une mention valorisante (produit de la ferme, produit à Haute Valeur Environnementale), les produits issus du commerce équitable ou de la pêche durable, et les produits bénéficiant du logo « Région Ultrapériphérique ».

5. Dans notre étude, le terme « acteur » renvoie parfois à un individu (par exemple, les personnes interrogées) et parfois à une organisation (par exemple, une ferme ou un restaurant). Dans la suite de l'article, nous préciserons le terme lorsque son utilisation pourrait prêter à confusion.

6. En France, les entreprises et les établissements publics ont le choix entre deux systèmes de restauration collective : la gestion directe et la gestion concédée. En gestion directe, la confection des repas et l'organisation du restaurant sont confiées à une équipe interne à l'entreprise. En gestion concédée, la confection des repas et l'organisation du restaurant sont déléguées à une 
société de services de restauration collective (par exemple, Sodexo, API, ELIOR, etc.). Ce prestataire est responsable juridiquement de la cantine.

7. L'expression renvoie à celle couramment utilisée par des associations ou des pouvoirs publics, de la fourche à la fourchette, pour évoquer et valoriser l'unicité et la traçabilité de la chaîne alimentaire. Ici nous ne partons pas de la production, mais de la consommation, d'où l'inversion de la formulation.

8. La démarche de labellisation $\mathrm{BBC}$ est présentée comme permettant la production de produits plus sains pour l'Homme et plus respectueux de l'environnement, grâce à l'introduction d'une alimentation de meilleure qualité pour les animaux d'élevage. Elle oriente les agriculteurs vers l'utilisation de graines oléo-protéagineuses locales (lin, lupin, féverole, luzerne, colza...) pour une alimentation animale plus riche en nutriments, et pour promouvoir une économie circulaire locale.

\section{RÉSUMÉS}

La relocalisation des systèmes alimentaires dans les territoires s'inscrit dans une volonté politique et citoyenne de reconnecter agriculture et alimentation, et de répondre aux enjeux du développement durable. Elle interpelle l'ergonomie, car une telle évolution ne peut s'entendre sans mutations du travail. Cet article repose sur une étude de cas exploratoire qui a été conduite sur le plateau de Saclay. L'étude concerne l'approvisionnement de cantines d'entreprise en produits locaux. Elle s'intéresse à des acteurs dont les statuts (responsable d'exploitation agricole, responsable de restaurant) et les expériences permettent de révéler une reconception progressive et "dans l'usage » des processus de coordination intra et inter-organisationnelles. L'étude de cas éclaire également l'élasticité de la définition territoriale saisie par ces acteurs impliqués dans le développement d'un système alimentaire local à l'aulne des valeurs sociales et culturelles qu'ils défendent. Ces acteurs s'approprient et modifient l'organisation d'un système qui traverse les frontières de leurs organisations. Nous proposons ici d'aborder le changement inter-organisationnel induit par la relocalisation de l'alimentation dans et par les territoires. Nous définissons ce changement comme un processus de conception collective mettant en dialogue deux processus de reconception des coordinations, l'un à l'échelle de l'organisation et l'autre à l'échelle des relations inter-organisationnelles. Finalement, nous caractérisons un «travail de territorialisation", singulier et situé, qui d'une part inclut ces activités de reconception des coordinations; et d'autre part vise à faire évoluer un espace géographique, culturel et/ou politique, que les acteurs identifient comme territoire, pour que cet espace corresponde à leur vision du local et du durable. Nous discutons des implications de ces résultats pour l'analyse et l'intervention ergonomiques.

The relocation of food systems in territories pursues both a political and civic ambition to reconnect agriculture and food, and to face the challenges of sustainable development. It calls upon ergonomics because such an evolution cannot be achieved without work transformations. The present article is based on an exploratory case study which was conducted on the ParisSaclay territory. The study concerns the supply of local products to staff canteens. It focuses on actors whose positions (farm manager, restaurant manager) and experiences reveal an ongoing or "practise-based" redesign of intra and inter-organisation coordination processes that aims to support the development of the local food system. The case study highlights the elasticity of the 
territorial definition experienced by the actors involved in the development of a local food system according to the social and cultural values they defend. The actors appropriate and change the organisation of a local food system which crosses the boundaries of their own organisations. In this article we propose to approach the inter-organisational change that results from the re-localization of food in and through the territories. We define such a change as a collective design process which creates a dialogue between two coordination re-design processes, one at the level of each organisation and the other at the level of inter-organisational relations. Finally, we define a unique and situated "territorialisation work" that includes the activities of the coordination re-design, and which aims to develop a geographical, cultural and/or political space that the actors identify as a territory, in order for this space to fit in with their vision of what local and sustainable food means. We discuss the implications of the results for both ergonomic analysis and intervention.

\section{INDEX}

Mots-clés : système alimentaire local, coordination inter-organisationnelle, coordination intraorganisationnelle, reconception organisationnelle, travail de territorialisation

Keywords : local food system, inter-organizational coordination, intra-organizational coordination, organizational design, territorialisation work

\section{AUTEURS}

\section{CHLOÉ LE BAIL}

Université Paris-Saclay, CNRS, UMR LISN, 91400, Orsay, France, chloe.le-bail@universite-parissaclay.fr

\section{MARIANNE CERF}

Université Paris-Saclay, INRAE, AgroParisTech, UMR SAD-APT, 75005, Paris, France, marianne.cerf@inrae.fr

\section{GWENOLA YANNOU-LE BRIS}

Université Paris-Saclay, INRAE, AgroParisTech, UMR SayFood, 91300, Massy, France, gwenola.yannou-lebris@agroparistech.fr 